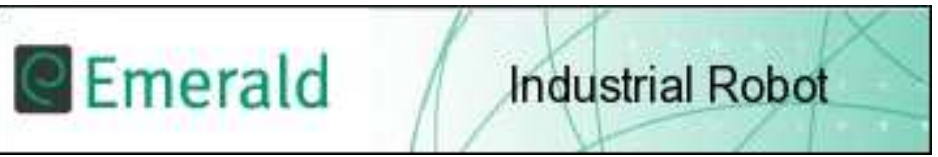

\title{
Analysis of the effects of time delay on the tele-operation of a mobile robot in various modes of operation
}

\begin{tabular}{|r|l|}
\hline Journal: & Industrial Robot: an International Journal \\
\hline Manuscript ID: & IR-08-726.R1 \\
\hline Manuscript Type: & Original Manuscript \\
\hline Keywords: & $\begin{array}{l}\text { Teleoperation < Industrial Robotics, Man machine interface (MMI) } \\
\text { Programming < Industrial Robotics, Joystick < Programming < } \\
\text { Industrial Robotics, Nuclear < Surveillance < Industries, Low cost } \\
\text { robots < Languages < Industrial Robotics, Ultrasonic < Sensor } \\
\text { Review }\end{array}$ \\
\hline
\end{tabular}




\section{ANALYSIS OF THE EFFECTS OF TIME DELAYS ON THE TELE-OPERATION OF A MOBILE ROBOT IN VARIOUS MODES OF OPERATION.}

\section{Structured Abstract}

Purpose: The effect of time delay on the ability of a human operator to complete a task with a tele-operated mobile-robot is investigated using two systems, two different ways of interacting with the mobile-robots and several different environments.

Design/methodology/approach: Tele-operators were observed completing a series of tasks using a joystick to control a mobile-robot while time delays were introduced to the system. They sat at a computer and viewed scenes remotely on a screen.

Cameras were either mounted on the robot or mounted externally so that they viewed both the environment and robot. Tele-operators completed the tests both with and without sensors. One robot system used an Umbilical Cable and one used a radio link.

Findings: In simple environments, a tele-operator may perform better without a sensor system to assist them but as time delays were introduced then there were more failures. In more complicated environments or when time delays were longer then teleoperators performed better with a sensor system to assist. Tele-operators may also tend to perform better with a radio link than with an umbilical connection.

Research limitations/implications: Tele-operated systems rely heavily on visual feedback and experienced operators. This paper investigates the effect of introducing a delay to the delivery of that visual feedback.

Practical implications: The paper suggests that in simple environments with short time delays then the amount of sensor support should be small but in more complicated environments or with longer delays then more sensor support needs to be provided.

Originality/value: Results from imposing time delays on a tele-operated mobile-robot are presented. Effects on the task of different ways of viewing activity on a computer display are presented, that is with cameras mounted on the robot or cameras mounted externally to view both the environment and robot. Results from using sensors to assist tele-operators are presented. The paper suggests that the amount of sensor support should be varied depending on circumstances.

Keywords: tele-operation, mobile robot, sensor, ultra-sonic. 


\section{Introduction}

This paper investigates the effects of time delays on the performance of tele-operated mobile robots. That performance is investigated using two tele-operated mobile-robot systems, two different ways of interacting with the mobile-robots and several different environments. One mobile-robot system used an umbilical cable and one used a radio transmitter and receiver. They had an ultrasonic sensor system that could be installed to assist the tele-operators.

Tele-operators were observed completing a series of tasks using a joystick to control a mobile-robot in two ways. Tele-operators sat in front of a computer screen and either viewed the display from a camera mounted in the environment to observe the robot or they viewed the scene ahead of the robot from a camera mounted on the robot. In each case the tele-operators completed tests both with and without the sensor system assisting them.

After recounting some background and a little about the sensor system and mobile-robot hardware, then the testing is explained and results are presented. The paper finishes with discussion, conclusions and suggestions for future work.

A main conclusion is that although in simple environments, a tele-operator may perform better without a sensor system to assist them (Sanders 1999a), but the failure rate increases significantly without the use of sensor systems, especially as time delays increased. In more complicated environments then tele-operators performed faster and with a better success to failure ratio when using a sensor system to assist. That effect became more pronounced as time delays increased. That builds on results from Milgram (1993) who showed that global performance can be improved by allowing each tele-operator to act within their own space.

Testing was within a built up areas and not over countryside terrain. That environment was made more complex for each set of experiments by increasing the number of obstacles and by adding sloping floors and sloping boundaries. Both an umbilical system and a radio controlled system were evaluated because umbilical systems are being considered for use in the Nuclear industry.

\section{Background}

Several tele-operation systems have been developed to allow human operators to execute tasks in remote or hazardous environments for a variety of applications (Sheriden, 1995) and some studies have recently been conducted to consider the way in which a tele-operator interacts with a mobile robot depending on various conditions (Sanders 2009a) but they did not consider time delays. 
When local tele-operation and remote slave sites are separated by some distance then time delays can be introduced. Any time delay can add distortion to commands and feedback (Fiorini, 1997) and reduce the performance of the system (Richard, 2003). Tele-operation systems tend to compromise between transparency and stability margins (Lawrence, 1993). Some control strategies for teleoperation systems with constant time delays have been proposed (Kim, 1992; Niemeyer, 1991) and for varying time delays (Niemeyer, 1998; Slawinski, 2006).

This paper investigates the effects of time delays on the tele-operatation of a mobile robot.

The real challenge in unstructured and difficult environments such as hazardous areas is primarily for mobile-robots (Luk et al, 2005; Rooks, 2006a; Sanders 2009c). Tele-robotics enables a robot to be remotely operated from outside a hazardous environment (Sands, 2006). Wheeled vehicles are considered in this paper because they are still the main mechanisms for moving over ground (Nakamura, 2008) despite finding it difficult to move freely over some terrain. Mobile-robots and unmanned vehicles are being increasingly used (and considered for future use) in nuclear plants (Luk, 2006a), for search and rescue (Kapoor, 2006; Marques, 2007; Molfino, 2007; Wang, 2007), security (Carnegie, 2004a \& b) and inspection (Love, 2007).

Tele-operated mobile-robots are generally directed along a path using manual controls and the master system has often been a joystick (Bergasa-Suso, 2005; Chester 2006 \& 2007; Dede, 2006; Goodwin, 1997; Sanders 1999 \& 2001a) although other input devices are available, for example a pointer (Sanders, 2001b, 2005 \& 2009b), switches (Stott 1995, 1997 and 2000a; Sanders, 2008a) or can be custom built, such as Virtual Reality interfaces (Stott, 2000b). Generally they are fitted with controllers that interface low current input devices to high current servo amplifiers, sometimes remotely through a radio connection or umbilical cable.

Much research has aimed to improve tele-operation and robotics for inspection and maintenance in hazardous or unpleasant environments (Sands, 2006), or in places where conventional techniques required cost intensive supporting infrastructures (Aguinaga, 2007; Eisinberg, 2007; Kochan, 2006; Li, 2007; Luk, 2006b; Zhang, 2007). At the time of writing, conventional robots are limited when a task requires a level of perception and decision making which cannot be met in a cost effective or robust way. 
For many applications, mobile-robots may not need autonomous control with geometric models of the environment (Sanders, 1995a \& 2009c). Instead a human operator may help a mobile-robot to explore environments. Other tasks may be best achieved with a wheeled base and on-board manipulator(s) (Luk, 2006b; Bakari, 2006), for example tasks involving many degrees of freedom, such as turning off a manual valve wheel. This could be achieved using a mobile base module with an arm module mounted on the base (Acaccia, 2008; Rooks, 2006b).

The way in which a human operator interacts with a mobile-robot can affect efficiency, and timecritical operations in emergencies require especially efficient human-machine interaction. This paper investigates that interaction using tele-operated mobile-robot systems with varying time delays.

Systems have tended to be open loop. Users have indicated a direction and the mobile-robot then moved in the required direction. Common disturbances include differences in mobile-robot wheels or tractors or their different reaction to surfaces and surface or gradient (Stott, 2000a; Sanders, 2008a; Urwin-Wright, 2002 \& 2003) as well as time delays. Users have been left to react to disturbances and correct trajectories.

Tele-operating systems are described in (DeJong, 2006). Automatic guided vehicles (AGVs) are described in Rocha et al (2007) and Tokhi (2007) described standardisation in mobile-robotics. Current challenges being faced in tele-operation are described in Chen et al (2007) and some seminal publications are Sheridan (1992 \& 2002). They are included here for reference and wider reading.

Two mobile-robot systems were used to investigate the effect of time delays on tele-operated systems; one with an Umbilical Cable and one with a radio transmitter and receiver. Both could have a sensor system installed.

\section{The mobile robot systems}

Sonar sensors are simple and have been widely used for mobile-robots (Gao, 2006). Ultrasonic ranging was selected, as it was simple and robust. A human user guided the tele-operated mobilerobot using a camera mounted on the mobile-robot or a camera to observe the local environment. Two $40 \mathrm{KHz}$ ultrasonic transmitter and receiver pairs could be mounted at the front of the mobilerobot. With suitable processing the ultrasonic image was converted to a simple representation of the environment. An integral function was used with joystick signals so that the tendency to turn when 
approaching an object could be over-ruled by the user, for example to align properly with another gap beyond the first.

The apparatus consisted of a parallel interface, which provided an input to a micro-computer, a dedicated controller with analogue interfacing, DC servo-amplifiers and joystick. A BobCat II base was modified to include the systems. This prototype system allowed mobile-robots to be driven under computer control by "fly-by-wire" (Sanders, 2001) to sense the environment and to modify control signals sent to the mobile-robot controller. The system is described in more detail in (Sanders, 2009a). The first mobile-robot system with an umbilical cable is shown in Figure 1.

\section{Figure 1 here Bobcat II Base Unit.}

The mobile-robot was electrically powered with a front wheel drive chassis and fibreglass body. The base was a heavy steel plate chassis to provide stability and rigidity. Two driven wheels were at the front and two trailing casters at the back. A camera could be mounted between the driving wheels in addition to the ultrasonic sensor pairs over each driving wheel. Trailing casters supported the rear of the base. Altering the differential of rotational speed of the driving wheels affected steering.

\subsection{Mobile-robot with an umbilical cable}

The direct link between the mobile-robot and joystick was severed and a computer processed control information. Sensors were activated and interrogated by the computer and the computer was programmed to modify the mobile-robot path. Alternatively, joystick control data could be processed and sent to the mobile-robot controller without modification. In this case the mobile-robot responded to joystick inputs as if it was an unmodified mobile-robot system. Software systems were constructed using methods discussed in (Sanders, 1993). Systems had three main levels: supervisory, strategic and servo control. These were similar to the levels described in (Sanders, 1995b; Tewkesbury, 1999a \& 1999b) and used in Sanders (1996, 2000 \& 2001c) and Tewkesbury (2001) and for the sensor systems discussed in Sanders (2008c).

Algorithms applied the following rules:

- The user remained in overall control.

- Systems only modified the trajectory of the mobile-robot when necessary.

- Movements of the mobile-robot were smooth and controlled. 
An imaginary potential field was generated around objects by the computer in response to information supplied by the sensor system (Chang, 2008; Khatib, 1986; Ko , 2006; Volpe, 1990). These algorithms assisted users if the mobile-robot was approaching an object and could collide.

\subsection{Mobile-robot system with a radio link}

A second test rig used a radio link instead of an umbilical cable. Most apparatus was re-used (mobile-robot base, sensor system, joystick, micro-computer, dedicated controller with analogue interfacing, DC servo-amplifiers, joystick and camera mounted between the driving wheels). The umbilical cable was replaced by a radio link and the parallel interface was replaced with a serial interface.

Communications between computer, mobile-robot controller, joystick and sensor system were all routed through an interface card and radio link. The Microcontroller Circuit was adjusted to receive serial information from the radio control receiver, calculate motor requirements and send information to actuate the motors. EMC testing was conducted with the radio link to avoid frequencies that might interfere with the controller, sensors and cameras.

\section{Testing}

Mobile-robot systems were tested in a laboratory and then in a variety of environments. The longest test runs were limited to just under 30 metres by the lengths of the umbilical cables used. The cables were up to 15 meters long and that allowed a distance of 15 metres out and back. Users quickly learned how the mobile-robot responded and learned to apply control signals earlier and to estimate stopping distance.

Tests were conducted to:

- Observe the operation of the system under joint computer and human control and compare that with human control alone for a variety of time delays.

- Record the number of successful tasks completed by tele-operators under various conditions and with various time delays.

- Record the number of failures with various time delays.

- Measure the improvement (if any) of using assistive systems when there are time delays. 
- Measure time taken by human tele-operators by themselves and then again with the assistance of sensor systems as gaps were slowly reduced in width and time delays increased.

- Measure the minimum gaps that human tele-operators were able to move through by themselves and then again with the assistance of sensor systems as time delays increased.

For each course, up to eight sets of tests took place. For each mobile-robot, four sets tests took place with out the sensor system or any automatic assistance and four with the sensor system providing automatic assistance. For each set of tests the tele-operators either viewed the scene ahead of the robot from a camera mounted on the robot or viewed the robot within the scene from a camera mounted to view the environment:

For each type of test, a standard obstacle course was set up in an environment. The environments used were:

\section{LABORATORY}

- Just two obstacles in a staggered formation and a constant open floor space with vertical walls around the edges.

\section{SIMPLE CORRIDORS}

- Flat surfaces and sloping surfaces.

- Corridor restricted with vertical walls but no doorways.

- Obstacles offset in a staggered formation.

\section{COMPLICATED CORRIDORS}

- Flat surfaces and sloping surfaces.

- Corridor restricted with vertical walls and doorways and with items on the walls (for example radiators and door surrounds).

- Doorways to pass through.

- More obstacles offset in a staggered formation.

\section{ENVIRONMENTS OUTSIDE}

- Complex environment with different flat and sloping surfaces.

- Bounded by different vertical and sloping edges.

- Objects in the environment as well as obstacles placed in the environment. 
All tele-operators were volunteers and came mainly from staff and students at the University of Portsmouth, and many were undergraduates or research students. Only seven of the 51 participants were not students. Participants received a clear explanation of the study (including risks and benefits of participation) and the University of Portsmouth Ethics Committee approved all experimental procedures. There were 40 males and 11 females. The 51 participants were aged 17-51 years (Mean: 24, Standard Deviation: 6).

Tele-operators were human beings and as such they were variable in their performance and so where possible, experiments were repeated several times.

For each of the series of tests, the tele-operators were allowed to repeat tests (with or without computers assisting them) as many times as they liked, or hours available allowed. That allowed them to learn the systems and to perform at their best in the time available and as time delays were increased. Testing was regarded as fun by participants and was popular. Competition was encouraged and people tried to beat their best in each test and tried to beat others at the same tests. In several cases, some people only managed to complete the tele-operation test or only managed to complete the computer assisted test and their results were discarded so that comparisons were only made between the same tele-operators.

The number of successful runs was recorded for each case along with the number of failures as time delays were increased. If a course was completed without any collision then that was counted as a success. If a collision occurred with anything (obstacle, wall, doorway etc) at any time during a test run then it was counted as a failure.

The first set of tests used the umbilical cable and was conducted to compare the ability of human tele-operators to move through a set course with gaps between obstacles set at a width of $88 \mathrm{~cm}$. That was $8 \mathrm{~cm}$ wider than the mobile-robot $(4 \mathrm{~cm}$ at each side). This was the compared with computer-assisted operation in a series of standard environments. If a smaller gap was achieved by any participant in one set of the tests then they made at least one attempt again at the other test to check that the result was not just due to learning the operation of the systems. If they then managed to get the robot to pass through smaller gaps then they made at least one attempt at the original test. Tests began at a pre-determined and constant start-position (and from a standing start) and widths were measured by two researchers using a ruler and a measure. If too few sets of results were recorded or if there were no pairs of results then results for that environment were discarded. 
A second set of tests was then conducted but using a radio link instead of an umbilical cable.

Figure 2 shows the two places where a delay could have been introduced before:

- displaying the camera view to the tele-operator $\left(h_{1}\right)$ after the joystick.

- transmitting the movement instructions to the mobile robot $\left(h_{2}\right)$.

The human operator observed the actual position $\left(x_{r}\right)$ and actual orientation $\left(f_{r}\right)$ of the mobile robot on a computer screen as position $\left(x_{1}\right)$ and orientation $\left(f_{1}\right)$ and a delay was introduced here. The velocity commands to the wheels $\left(v_{1}\right)$ could also have been delayed so that a delayed signal $\left(v_{r}\right)$ was actually applied to the mobile robot. Both these communication channels could have introduced a time delay of $h$ that was composed of a forward delay $h_{2}$ (from the local site to the remote site) and a backward delay $h_{1}$ (from the remote site to the local site).

In the event, only delay $h_{1}$ was used to provide a delay from the remote site to the local site. This was achieved by delaying the display of pictures of the scene in the computer being used by the teleoperator to view the situation. This was selected as it was the simpler of the two delays to introduce.

Figure 2 here Block diagram of the tele-operation system showing potential delays that could have been built in. (Reproduced from Slawinski, 2006)

Figure 3 shows a diagram of Complicated Corridor 3. The arrows show the general route taken by the mobile robot and the shaded blocks show the positions of the obstacles placed in the path of the robot. Complicated Corridor 3 also included two double doorways to pass through. In both cases, one of the doors was held open and the other was shut so that the mobile robot needed to zig zag through them..

Figure 3 here A diagram of Complicated Corridor 1.

Figure 4 shows a tele-operator in a laboratory controlling the robot moving through an outside environment by using an Ethernet connection to an outside wall and then an umbilical cable to control the robot. A camera is observing the environment and the robot and displaying the scene on a computer screen. The tele-operator is using the joystick to guide the robot. 
Figure 4 here $A$ tele-operator in a laboratory controlling the robot through an umbilical cable with a camera set up to observe the environment and the robot.

Figure 5 shows the robot moving though a complicated corridor and being controlled via a radio connection. The tele-operator in a laboratory is being assisted by the sensor system on the mobilerobot. The sequence shows the one successful run competed with a delay of 2.5 seconds.

Figure 5 here Radio controlled robot moving though a complicated corridor assisted by the sensor system on the mobile-robot.

Figure 6 shows another tele-operator in a laboratory controlling the robot using a radio connection to control the robot.

Figure 6 here $A$ tele-operator in a laboratory controlling the mobile-robot through a radio link using a camera mounted between the driving wheels.

A camera is mounted on the robot and is observing the scene ahead of the robot and displaying the scene on a computer screen. The tele-operator is using the joystick to guide the robot through an outdoor course.

Figure 7 shows the scene from a camera mounted on the front of the robot as it moves though Complicated Corridor 2. The mobile-robot is being controlled via the umbilical cable. A tele-operator in a laboratory is guiding the mobile-robot assisted by the sensor system on the mobile-robot. A researcher with a laboratory digital clock can be seen at the end of the course and another researcher was following the mobile-robot with a stop watch. The sequence of images shows the sequence observed by the tel-operator for the one successful completion with a delay of 3 seconds.

Figure 7 here View from a camera mounted on the robot connected via an umbilical cable and moving though a complicated corridor while being assisted by the sensor system. 
Figure 8 shows the mobile-robot moving though a complicated outdoor environment and being controlled via an umbilical cable. The tele-operator in a laboratory is being assisted by the sensor system on the mobile-robot and the time delay was set to half of a second.

Figure 8 here View observed by a tele-operator in a laboratory from an external camera while driving the robot though a complicated outdoor environment using an umbilical cable and assisted by the sensor system with a time delay of 0.5 seconds.

Figure 9 shows the view from a camera mounted on the front of the mobile-robot as it moves though the first part of the same outdoor environment with a delay of one second. In this case the robot is being controlled via an umbilical cable and the tele-operator in a laboratory is not being assisted by the sensor system on the mobile-robot.

Figure 9 here View from a camera mounted on the front of the mobile-robot moving though the first part of a complicated outdoor environment. With a delay of one second

\section{Results.}

The mobile-robot successfully negotiated obstacles in various set courses during testing. Assistive computer systems allowed automatic recovery from collision courses. Some chaotic factors existed. For example, trailing casters could throw the mobile-robot off-line and variation in floor surface, slope or wheel position could affect results.

\subsection{Performance with and without the ultrasonic sensor systems.}

Figure 10 shows the average of the best times taken for a mobile robot with an umbilical cable to complete various set courses while the tele-operators watched the scene ahead of the mobile robot using a camera ounted on the robot. The graph on the left shows the results using the umbnilical cable and the graph on the right shows results using the radio link. The vertical scale is the average time taken in seconds to complete successful tests. In the simple environments (shown on the left of each graph; laboratory and empty corridors), tele-operators completed tasks more quickly without any aid from computer and sensor systems. In the more complicated environments (shown on the right of 
the graph; complicated corridor and outside), tele-operators completed tasks more quickly with the aid of computer and sensor systems. The lower two graphs show the average of the best times taken when a delay of one second was introduced. That form of results was repeated when a camera was mounted onto the mobile-robot and when a camera was mounted in the environment so that the mobile-robot and the environment were visible to the user on a computer screen.

Figure 10 here Average of the best times taken to complete tests when a camera was mounted on the mobile-robot (top) and with a time delay of one second introduced (bottom) and with the umbilical cable (left) and the radio link (right).

As the gaps between obstacles on the courses were reduced (by $0.5 \mathrm{~cm}$ each time) then the smallest gap successfully achieved by each tele-operator was recorded along with the number of successful and failed attempts. The tele-operators consistently managed to complete set courses with through smaller gaps when they were using the sensor system. That form of the results was repeated in all of the environments and the results for the system using the umbilical cable and repeated again when the umbilical cable was replaced with a radio link.

The average improvement in $\mathrm{cm}$ achieved by the sensor system for the various set courses was considered and some results are shown in figure 11 . The results shown are for test with the camera mounted on the mobile-robot. Tests with the umbilical cable are shown on the left and for the radio link on the right. The two graphs at the top are without an time delay and the two graphs at the bottom are with a delay of one second. Trend lines are also included.

Figure 11 here Results from tests of varying the width with a time delay of one second introduced (bottom) and with the umbilical cable (left) and the radio link (right) when the camera was mounted on the mobile-robot.

As the environments became more complicated (or the gaps were made smaller) then the human operators found it more difficult to judge the width of the gaps or the successful trajectory of the mobile-robot to pass through those gaps. The human tele-operators had to rely more and more on the sensor systems. Human tele-operators consistently passed through smaller gaps when being assisted by the sensors and computer systems. Different surfaces, slopes and boundaries tended to turn robots and sensors became most useful in steering through gaps in those cases. The automated systems managed to consistently correct the trajectory of the mobile-robot to a repeatable standard. 
Results became more pronounced as human operators were removed from immersion within the situation and environment and as time delays increased.

When human tele-operators were made to control the mobile-robot via a camera watching both the robot and environment then they found passing though gaps more difficult. With the assistance of sensor systems then mobile-robots could make it faster through smaller gaps or when time delays increased (providing the human tele-operator had lined up the mobile-robot sufficiently).

\subsection{Comparison between the umbilical cable and radio link}

In the environments tested, tele-operators tended to perform better with the radio link compared to the umbilical cable. Figure 12 show the results from testing in Simple Corridor 2 (with and without the assistance of the computer and sensor systems). In almost every case, tele-operators performed better with the sensor systems as the time delay increased but that effect became more significant as the delay became longer.

\subsection{Average times taken to complete courses with the narrowest gaps}

As the gaps between obstacles on the courses were reduced, the fastest times taken to complete the courses were recorded for each tele-operator as the time delays were increased. Figure 13 shows the average of the best times taken to complete Simple Corridor 2 with the minimum gap widths and with a delay of one second. The lines marked with crosses are with the sensor systems engaged and the lines marked with diamonds are without any sensor systems.

As the gaps were reduced in width, then initially the tele-operators completed the courses more quickly without the sensor systems engaged and as the gaps became smaller then the tele-operators competed the tests more quickly with the sensor systems engaged.

Figure 12 here Results from testing in Simple Corridor 2 as the time delay was increased from real time (no delay) to 2.5 seconds in 0.5 second increments.

Figure 13 here Average of the fastest times taken with reducing gap width between obstacles in Simple Corridor 2 with a time delay of one second introduced (below) for a tele-operator with a camera mounted on the mobile robot (left) and with a camera viewing both the environment and the mobile robot (right). 


\subsection{Failure rates when completing courses with the narrowest gaps}

Figures 14 to 15 show results from recording the successful and failed attempts both with and without the sensor system to assist when using a camera mounted on the robot and a camera observing the scene, for both the system with an umbilical cable and the system with a radio link were tested. The $x$ axis for each chart is the different environments.

The average number of successful attempts and the average number of failed attempts is shown on the left of each figure. The centre bar chart in each figure shows the percentage of failed attempts compared to total attempts. The right hand bar chart shows the difference between the number percentage failures between using a sensor system and not. The top three bar charts are without any time delay and the bottom three are woth a one second delay.

Figure 14 here Comparison between average number of successful and average number of failed attempts with a time delay of one second introduced (below) while using a camera mounted on the robot and using an umbilical cable (left), percentage of failed attempts while using a camera mounted on the robot and using an umbilical cable (centre) and difference between the percentage of failed attempts with and without the sensor system while using a camera mounted on the robot and using an umbilical cable

Figure 15 here Comparison between average number of successful and average number of failed attempts with a time delay of one second introduced (below) while using a camera mounted on the robot and using a radio link (left), percentage of failed attempts while using a camera to observe the robot and using a radio link (centre) and difference between the percentage of failed attempts with and without the sensor while usng a radio link (right).

\section{Discussion and conclusions}

The Student's t-test was used to compare means of samples. From each sample, the average (mean) $\bar{x}$ was calculated with a measure of dispersion (range of variation) of data around the sample mean (variance $S^{2}$ ) and thence the standard deviation (S). Having obtained those values, they were then used to estimate population mean $\mu$ and variance $\sigma^{2}$.

The individual sets of tests were not all statistically significant so that caution was required before generalising the results. 
Because pairs of tests and results took place, then it was possible to use a paired-samples statistical test. Results were arranged into two sets of replicate data; pairs of results with and without sensor assistance. The paired samples test was used because people (tele-operators) were inherently variable. Pairing removed much of that random variability as the same tele-operators had completed both sets of tests. When results were analysed using a paired-samples statistical test then results were statistically significant. The paired-samples statistical test shows the use without a sensor system and with a sensor system to be significantly different at $p<0.05(95 \%$ probability that this result would not occur by chance alone).

In simple environments, tele-operators performed faster without a sensor system to assist them but in more complicated environments or with longer time delays then tele-operators performed faster with a sensor system to assist them. Results show that with wide gaps between obstacles or in simple environments then human tele-operators consistently performed set tasks more quickly without any assistance from the computer systems and sensors. As gaps between obstacles were reduced or environments became more complicated as time delays were increased then human operators could not judge gap widths or the successful trajectory of the mobile-robot to pass through those gaps. When the environment became more complicated then tele-operators consistently performed better with the assistance of the sensors and computer systems.

As gaps became reduced in width, using assistive systems consistently performed faster than human tele-operators alone. Results tended to become more pronounced as human operators were removed from immersion within the situation and as the delays increased.

Tele-operators tended to perform better with a radio link than with an umbilical connection because the umbilical connections sometimes affected mobile-robot steering (especially when reversing or when mobile-robots turned back on themselves). Radio connection had the advantage of being more manoeuvrable.

Tele-operators sometimes performed better with a camera mounted on the robot and looking ahead compared with pre-mounted cameras observing robots and environment but that type of result appeared to depend on the specific task.

When a robot turned back on itself during a task (for example changing from coming towards the camera to moving away from the camera) then joystick controls effectively reversed as the camera 
still viewed the robot and environment from the same place. Tele-operators found that difficult and that effect appears to have accounted for at least some of the difference.

A delay could have been introduced in two places but it was only introduced in displaying the camera view to the tele-operator. The system needs to be retested with a delay after the joystick and before transmitting the movement instructions to the mobile robot as results may then be significantly different. In any real system a delay would probably be present in both if it was present in one.

An implication of the results for tele-operated systems is that sensors should not be used in freely navigable regions with good views but should be reseraved for restricted areas with poor views.

\section{Future work}

Results from the work need to be investigated more fully. Further statistical analysis could take place using the existing paired-samples but further testing would make data more accurate.

A cable drum system could be included for the umbilical cable so that the cable could be retracted when the mobile robot was returning to the start and force feedback (Sanders, 2007; Shirinov, 2004; Song, 2006) could be used to create a haptic joystick.

Delay compensation could be placed on the local and remote sites of the teleoperation system. A control structure could be introduced to improve the stability of the delayed system, possibly using a model of the mobile robot and a dynamic model of the human operator. Intelligence (Sanders, 2008) could be built into the system. 


\section{References}

Acaccia G, Bruzzone L and Razzoli R (2008). A modular robotic system for industrial Applications. Assembly Automation 28 (2), pp151-162.

Aguinaga I, Borro D and Matey L (2007). Path-planning techniques for the simulation of disassembly tasks, Assembly Automation, 27 (3), pp 207-214.

Anderson RJ and Spong M (1989). Bilateral control of teleoperators with time delay," IEEE Trans. Autom. Control 34(5), 494-501 (1989).

Bakari MJ and Seward DW (2006). Human arm-like mechanical manipulator - the design and development of a multi-arm mobile robot for nuclear decommissioning. ICINCO 2006, Proc' 3rd Int, Conf Informatics in control, automation and robotics - Robotics and Automation, pp 168-175. ISBN: 972-8865-60-0

Bergasa-Suso J, Sanders DA, Tewkesbury GE (2005). Intelligent browser-based systems to assist Internet users. IEEE Transactions on Education 48 (4), pp 580-585.

Carnegie DA and Cordes JC (2004a). The mechanical design and construction of a mobile outdoor multiterrain robot. Proc' IMechE Part B - Jnl Eng Man, 218 (11), pp 1563-1575.

Carnegie DA, Loughnane DL and Hurd SA (2004b). The design of a mobile autonomous robot for indoor security applications. Proc' IMechE Part B - Jnl Eng Man, 218 (5), pp 533-543.

Chang YC and Yamamoto Y (2008). On-line path planning strategy integrated with collision and dead-lock avoidance schemes for wheeled mobile robot in indoor environments. Ind Rob: An int' jnl, 35 (5), pp 421-434.

Chen JYC, Haas EC and Barnes MJ (2007). Human Performance Issues and User Interface Design for Teleoperated Robots. IEEE Transactions on Systems, Man, and Cybernetics, Part C: Applications and Reviews, 37, pp 1231-1245.

Chester S, Tewkesbury G, Sanders D, et al. (2007). New electronic multi-media assessment system. Web Information Systems and Technologies, Volume: 1, pp 414-420.

Chester S, Tewkesbury G, Sanders D, et al. (2006). New electronic multi-media assessment system. WEBIST 2006: Proceedings of the Second International Conference on Web Information Systems and Technologies, pp 320-324.

Dede $M$ and Tosunoglu S (2006). Fault-tolerant teleoperation systems design. Ind Rob: An int' jnl, 33 (5), pp 365-372.

DeJong BP, Faulring EL, Edward Colgate J, Peshkin MA, Kang H, Park YS and Ewing TF (2006). Lessons learned from a novel tele-operation test-bed. Ind Rob: An Int Jnl, Vol 33, No 3, pp 187 - 193. ISSN: 0143991X.

Eisinberg A, Menciassi A, Dario P, Seyfried J, Estana R and Woern H (2007). Teleoperated assembly of a micro-lens system by means of a micro-manipulation workstation. Assembly Automation, 27 (2), pp 123-133.

Fiorini P and Oboe R (1997). Internet-based telerobotics: Problems and approaches," Proceedings of the ICAR'97, Monterey, CA (July 7-9, 1997), pp. 765-770. Teleoperation of mobile robots with delay 681

Gao W and Hinders M (2006). Mobile robot sonar backscatter algorithm for automatically distinguishing walls, fences, and hedges. Int Jnl of Robotics Research 25 (2), pp 135-145.

Goodwin MJ, Sanders DA, Poland GA et al. (1997). Navigational assistance for disabled wheelchair-users. Journal of systems architecture, 43 (1-5), pp 73-79. 


\section{Industrial Robot}

Kapoor C and Tesar D (2006). Integrated teleoperation and automation for nuclear facility cleanup. Ind Rob: An int' jnl, 33 (6), pp 469-484.

Kim W, Hannaford B and Bejczy A (1992). Force reflection and shared compliant control in operating telemanipulators with time delay," IEEE Trans. Robot. Autom. 8(2), 176-185.

Khatib, O (1986), "Real-time obstacle avoidance for manipulators and mobile robots", Proc of IEEE Int. Conf. on Robotics and Automation, Vol. 5 No.1, pp.90-8.

Ko JP and Lee JM (2006). Tactile tele-operation of a mobile robot with a collision vector. ROBOTICA 24, pp 11-21.

Kochan A, (2006). CLAWAR highlights research progress on climbing and walking robots. Ind Rob: An int' jnl, 33 (2), pp 112-116.

Lawrence DA. (1993). Stability and transparency in bilateral teleoperation," IEEE Trans. Robot. Autom. 9 (5).

Li HC, Gao HM and Wu L (2007). Teleteaching approach for sensor-based arc welding telerobotic system. Ind Rob: An int' jnl, 34 (5), pp 423-429.

Love P. Kalra, Jason Gu (2007). An autonomous self contained wall climbing robot for non-destructive inspection of above-ground storage tanks. Ind Rob: An Int' Jnl, Vol 34, No 2, pp 122 - 127. ISSN: 0143-991X.

Luk BL, Cooke DS, Galt S, Collie AA and Chen S (2005), "Intelligent legged climbing service robot for remote maintenance applications in hazardous environments", Journal of Robotics and Autonomous Systems, Vol. 53/2, pp. 142-52.

Luk BL, Collie AA, Cooke DS and Chen S. (2006a). Walking and climbing service robots for safety inspection of nuclear reactor pressure vessels. Measurement \& Control 39 (2), pp 43-47.

Luk BL, Liu KP, Collie AA, Cooke DS and Chen S (2006b). Tele-operated climbing and mobile service robots for remote inspection and maintenance in nuclear industry. Ind Rob: An Int' Jnl. Vol 33, No 3, pp $194-204$. ISSN: 0143-991X.

Marques C, o Cristo J, Alvito P, Lima P, o Fraza J, Ribeiro I and Ventura R (2007). A search and rescue robot with tele-operated tether docking system. Ind Rob: An int' jnl, 34 (4), pp 332-338

Milgram P. Zhai S, Drascic D and Grodski J (2003). Applications of augmented reality for human-robot communication. Proceedings of the 1993 IEEE / RSJ International Conference on Intelligent Robots and Systems 93 (IROS 93), Volume 3, Issue, 26-30 pp 1467 - 1472.

Molfino R, Zoppi M and Rimassa L (2007). Rescue robot module with sliding membrane locomotion. Ind Rob: An int' jnl, 35 (3), pp 211-216.

Nakamura T and Satoh K (2008) Development of an omni-directional mobile robot using traveling waves based on snail locomotion. Ind Rob: An int' jnl, 35 (3), pp 206-210.

Niemeyer G and Slotine JJ (1991). Stable adaptive teleoperation. IEEE J. Ocean. Eng. 16(1), 152-162 (1991).

Niemeyer G and Slotine JJ (1998). Towards force reflecting teleoperation over the Internet," Proceedings of the IEEE International Conference on Robotics and Automation, Leuven, Belgium (ICRA'98), pp. 1909-1915.

Richard JP (2003). Time-delay systems: An overview of some recent advances and open problems," Automatica 39, pp 1667-1694.

Rooks B (2006a). Plotting future UK robotics research Programmes. Ind Rob: An int' jnl, 33 (3), pp 165-169. 
Rooks B (2006b). The harmonious robot. Ind Rob: An int' jnl, 33 (2), pp 125-130.

1

Rocha R, Cunha A, Varandas J, Dias J (2007). Towards a new mobility concept for cities: architecture and programming of semi-autonomous electric vehicles. Ind Rob: An Int' Jnl. Vol 34, No 2, pp $142-149$.

Sanders DA (1993). System specification II. Microprocessing and microprogramming, 38 (1-5), p 833.

Sanders DA (1995a), "Real time geometric modelling using models in an actuator space and Cartesian space", Jnl of Robotic Systems, J. Wiley, Vol. 12 No.1, pp.19-28.

Sanders DA (1995b), "The modification of pre-planned manipulator paths to improve the gross motions associated with the pick and place task", ROBOTICA, Int. Jnl of Information, Education \& Research in Robotics \& Al, Vol. 13 pp.77-85.

Sanders DA, Haynes BP, Tewkesbury GE, et al (1996). The addition of neural networks to the inner feedback path in order to improve on the use of pre-trained feed forward estimators. Mathematics and computers in simulation 41 (5-6), pp 461-472.

Sanders DA (1999a). Perception in robotics. Industrial Robot, 26 (2), pp 90-92.

Sanders DA and Stott IJ (1999) A new prototype intelligent mobility system to assist powered wheelchair users. Ind' Robot; an int' jnl, Vol 26, No 6, pp 466-475.

Sanders DA and Hudson, AD (2000). A specific blackboard expert system to simulate and automate the design of high recirculation airlift reactors. Mathematics and computers in simulation 53 (1-2), pp 41-65.

Sanders DA and Baldwin A (2001a). X-by-wire technology. Total Vehicle Technology: Challenging current thinking, pp 3-12.

Sanders DA and Rasol Z (2001b) An automatic system for simple spot welding tasks Total Vehicle Technology: Challenging current thinking, pp 263-272.

Sanders DA, Cawte $\mathrm{H}$ and Hudson AD (2001c). Modelling of the fluid dynamic processes in a highrecirculation airlift reactor. Int Jnl of energy research 25 (6), pp 487-500.

Sanders DA, Urwin-Wright SD, Tewkesbury GE, et al (2005). Pointer device for thin-film transistor and cathode ray tube computer screens. Electronics Letters, 41 (16), pp 894-896.

Sanders DA (2007). Force sensing. Ind Rob -an int' jnl: 34 (4), pp 268-268.

Sanders DA (2008a). Controlling the direction of "walkie" type forklifts and pallet jacks on sloping ground. Assembly Automation, 28 (4), pp 317-324.

Sanders DA (2008b). Progress in Machine Intelligence Industrial Robot - An International Journal Volume: 35 Issue: 6, Pages: 485-487.

Sanders DA (2008c). Environmental sensors and networks of sensors. Sensor Review 28 (4), pp 273-274.

Sanders DA (2009a). Comparing speed to complete progressively more difficult mobile robot paths between human tele-operators and humans with sensor systems to assist. Assembly Automation. AA-08-057.

Sanders DA (2009b). A pointer device for TFT display screens that determines position by detecting colours on the display using a colour sensor and an Artificial Neural Network. Displays. DISPLA-D-08-00006.

Sanders DA (2009c). Recognizing shipbuilding parts using artificial neural networks and Fourier descriptors. Proc. IMechE, Part B: J. Engineering Manufacture, 223(B3), 337-342. 
Sands D (2006). Cost effective robotics in the nuclear industry . Ind Rob: An Int' Jnl. Vol 33, No 3, pp 170 173. ISSN: 0143-991X.

Sheridan T (1992). Telerobotics, automation, and human supervisory control. Cambridge, MA: MIT Press.

Sheridan TB (1995). Teleoperation, telerobotics and telepresence: A progress report," Control Eng. Pract. 3(2), pp 205-214.

Sheridan T (2002). Humans and Automation: System Design and Research Issues. John Wiley and Sons.

Shirinov A, Kamenik J and Fatikow S (2004). Haptic interface for a nanohandling robot. Assembly Automation, Volume $24 \cdot$ Number 1, pp. 78-87.

Slawinski E, Mut V and Postigo JF (2006). Teleoperation of mobile robots with time-varying delay. Robotica (2006) volume 24, pp. 673-681.

Song G, Guo SX, Wang Q (2006). A tele-operation system based on haptic feedback. IEEE Int' Conf' on Information Acquisition, Peoples R China, Vols 1 and 2, pp 1127-1131.

Stott IJ, Sanders DA and Goodwin MJ (1995). Improvements in real time high-level micro computer control of a wheelchair using sensor systems. IEEE Proceedings of Euromicro, pp 335-340.

Stott IJ, Sanders DA and Goodwin MJ (1997). A software algorithm for the intelligent mixing of inputs to a teleoperated vehicle. Jnl of Systems Architecture 43 (1-5), pp 67-72.

Stott IJ and Sanders DA (2000a). New powered wheelchair systems for the rehabilitation of some severely disabled users. Int Jnl of Rehabilitation Research 23 (3), pp 149-153.

Stott IJ and Sanders DA (2000b). The use of virtual reality to train powered wheelchair users and test new wheelchair systems. Int Jnl of Rehabilitation Research 23 (4), pp 321-326.

Tewkesbury GE and Sanders DA (1999a). A new simulation based robot command library applied to three robots. Jnl of Robotic Systems 16 (8), pp 461-469.

Tewkesbury GE and Sanders DA (1999b). A new robot command library which includes simulation. $2 \mathrm{Jnl}$ of Robotic Systems 26 (1), pp 39-48.

Tewkesbury GE and Sanders, DA (2001). The use of distributed intelligence within advanced production machinery for design applications. Total vehicle technology: challenging current thinking pp 255-262.

Urwin-Wright S, Sanders D, Chen S (2003). Predicting terrain contours using a feed-forward neural network. Engineering applications of Al, 16 (5-6), pp465-472.

Urwin-Wright, S; Sanders, D; Chen, S (2002). Terrain prediction for an eight-legged robot. Journal of robotic systems, 19 (2), pp 91-98.

Tokhi MO (2007). Viewpoint - Mobile robotics moves forward on standardisation. Ind Rob: An Int' Jnl. Vol 34 , No 2.

Volpe R. and Khosla P. (1990), "Manipulator control with superquadric artificial potential functions: theory and experiments", IEEE Transactions on Systems, Man and Cybernetics, Vol. 20 No. 6, pp. 1423-36.

Wang $Z$ and Hong Gu (2007). A review of locomotion mechanisms of urban search and rescue robot Ind Rob: An int' jnl, 34 (5), pp 400-411.

Zhang H, Zhang J, Wang W, Liu R, Zong G (2007). A series of pneumatic glass-wall cleaning robots for highrise buildings. Ind Rob: An Int' Jnl. Vol 34, No 2 Page: 150 - 160. 
2

3

4

5

6

7

8

9

10

11

12

13

14

15

16

17

18

19

20

21

22

23

24

25

26

27

28

29

30

34

35

36

37

38

39

40

41

42

43

44

45

46

47

48

49

50

51

52

53

54

55

56

57

58

59

60 \\ Page 21 of 38}

Industrial Robot

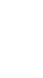

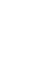

.

(n)

(15

(2)

0

(n)

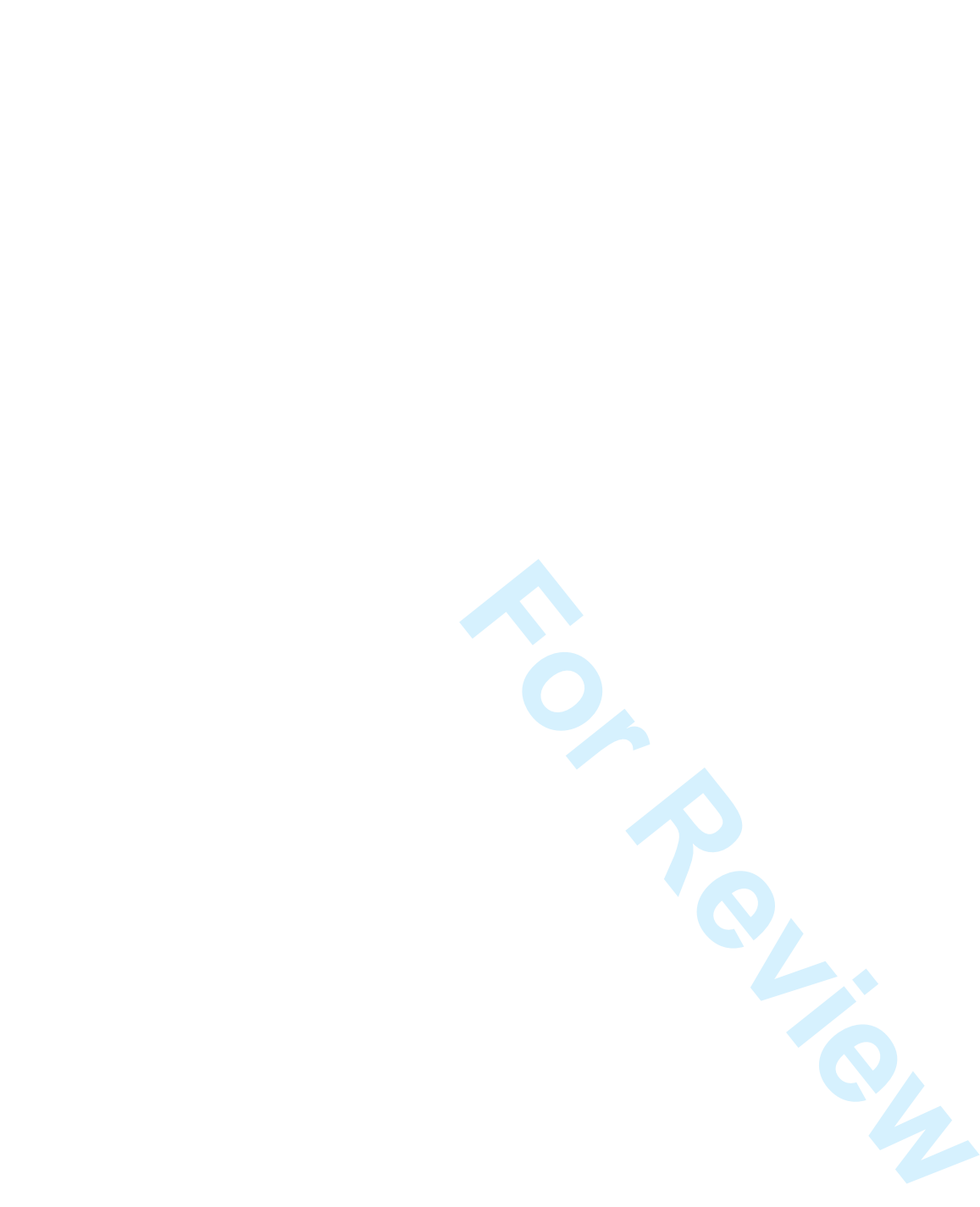

(2)


Figure 1 Bobcat II Base Unit.

Figure 2 Block diagram of the tele-operation system showing potential delays that could have been built in.

Figure 3 A diagram of Complicated Corridor 1.

Figure $4 \mathrm{~A}$ tele-operator in a laboratory controlling the robot through an umbilical cable with a camera set up to observe the environment and the robot.

Figure 5 Radio controlled robot moving though a complicated corridor assisted by the sensor system on the mobile-robot.

Figure 6 A tele-operator in a laboratory controlling the mobile-robot through a radio link using a camera mounted between the driving wheels.

Figure 7 View from a camera mounted on the robot connected via an umbilical cable and moving though a complicated corridor while being assisted by the sensor system.

Figure 8 View observed by a tele-operator in a laboratory from an external camera while driving the robot though a complicated outdoor environment using an umbilical cable and assisted by the sensor system with a time delay of 0.5 seconds.

Figure 9 View from a camera mounted on the front of the mobile-robot moving though the first part of a complicated outdoor environment. With a delay of one second

Figure 10 Average of the best times taken to complete tests when a camera was mounted on the mobile-robot (top) and with a time delay of one second introduced (bottom) and with the umbilical cable (left) and the radio link (right).

Figure 11 Results from tests of varying the width with a time delay of one second introduced (bottom) and with the umbilical cable (left) and the radio link (right) when the camera was mounted on the mobile-robot. 
Figure 12 Results from testing in Simple Corridor 2 as the time delay was increased from real time (no delay) to 2.5 seconds in 0.5 second increments.

Figure 13 Average of the fastest times taken with reducing gap width between obstacles in Simple Corridor 2 with a time delay of one second introduced (below) for a tele-operator with a camera mounted on the mobile robot (left) and with a camera viewing both the environment and the mobile robot (right).

Figure 14 Comparison between average number of successful and average number of failed attempts with a time delay of one second introduced (below) while using a camera mounted on the robot and using an umbilical cable (left), percentage of failed attempts while using a camera mounted on the robot and using an umbilical cable (centre) and difference between the percentage of failed attempts with and without the sensor system while using a camera mounted on the robot and using an umbilical cable

Figure 15 Comparison between average number of successful and average number of failed attempts with a time delay of one second introduced (below) while using a camera mounted on the robot and using a radio link (left), percentage of failed attempts while using a camera to observe the robot and using a radio link (centre) and difference between the percentage of failed attempts with and without the sensor while usng a radio link (right). 
Figures

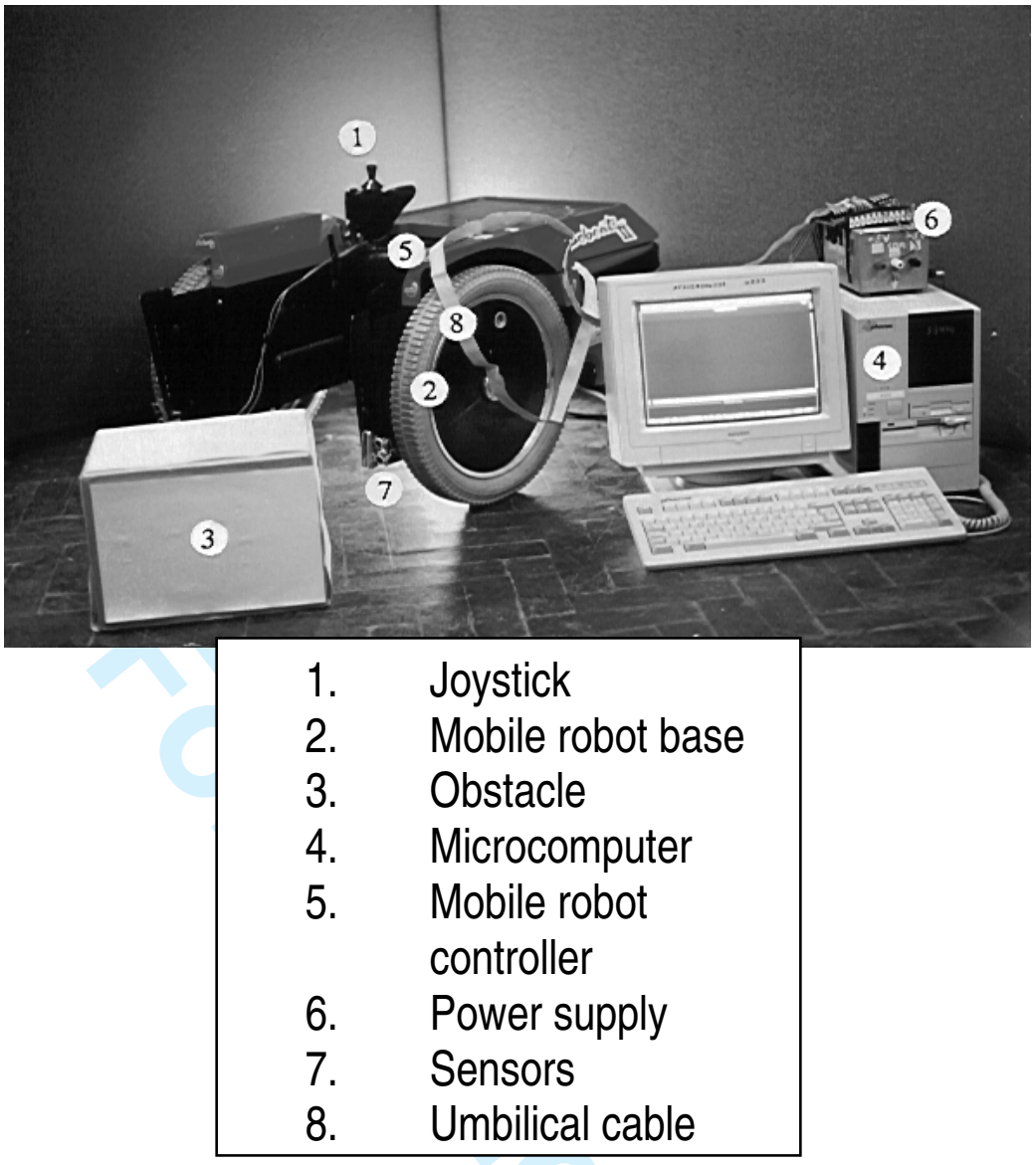

Figure 1 Bobcat II Base Unit. 


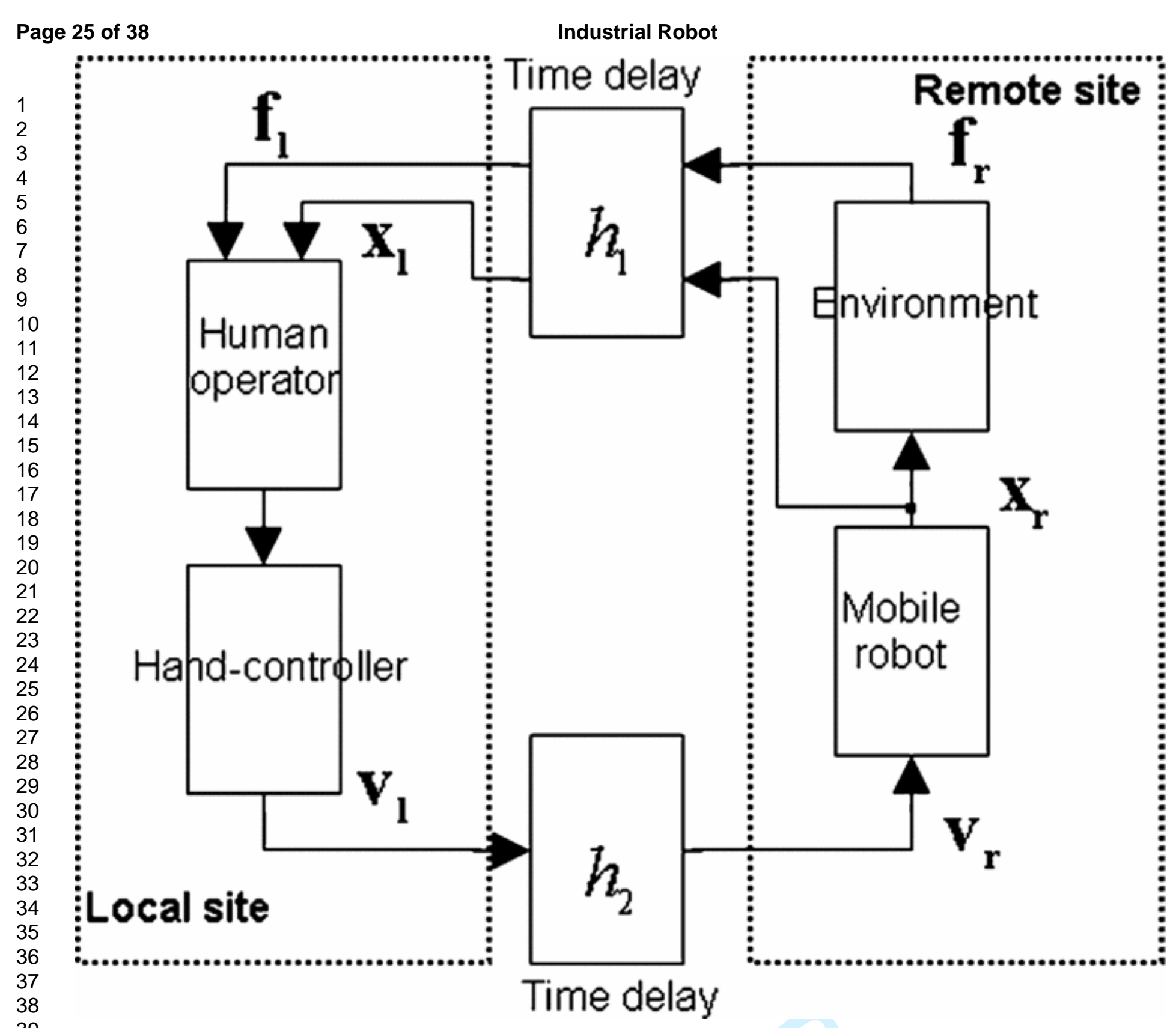

Figure 2 Block diagram of the tele-operation system showing potential delays that could have been built in.

(Reproduced from Slawinski, 2006) 


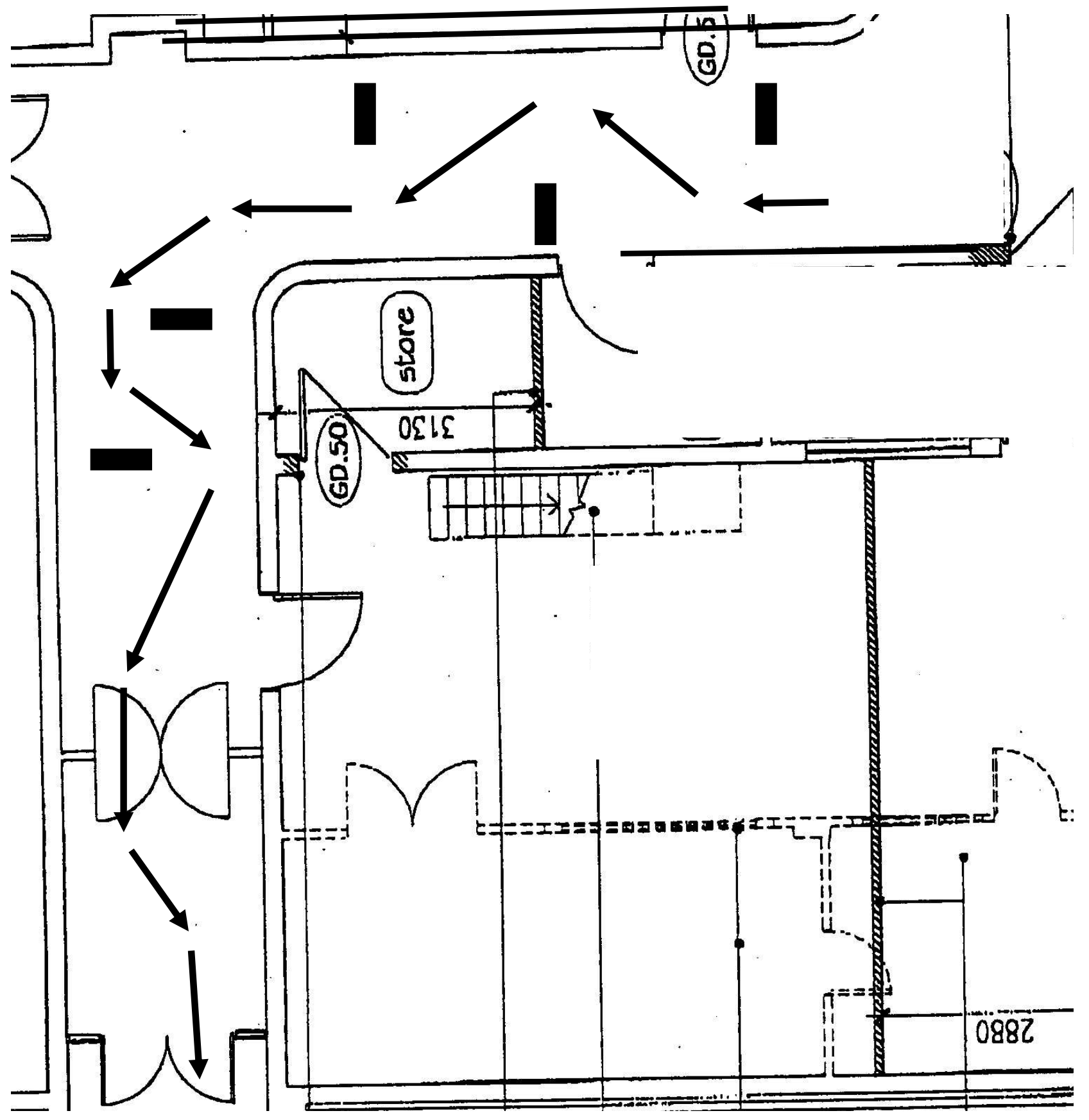

Figure 2 A diagram of Complicated Corridor 1. 


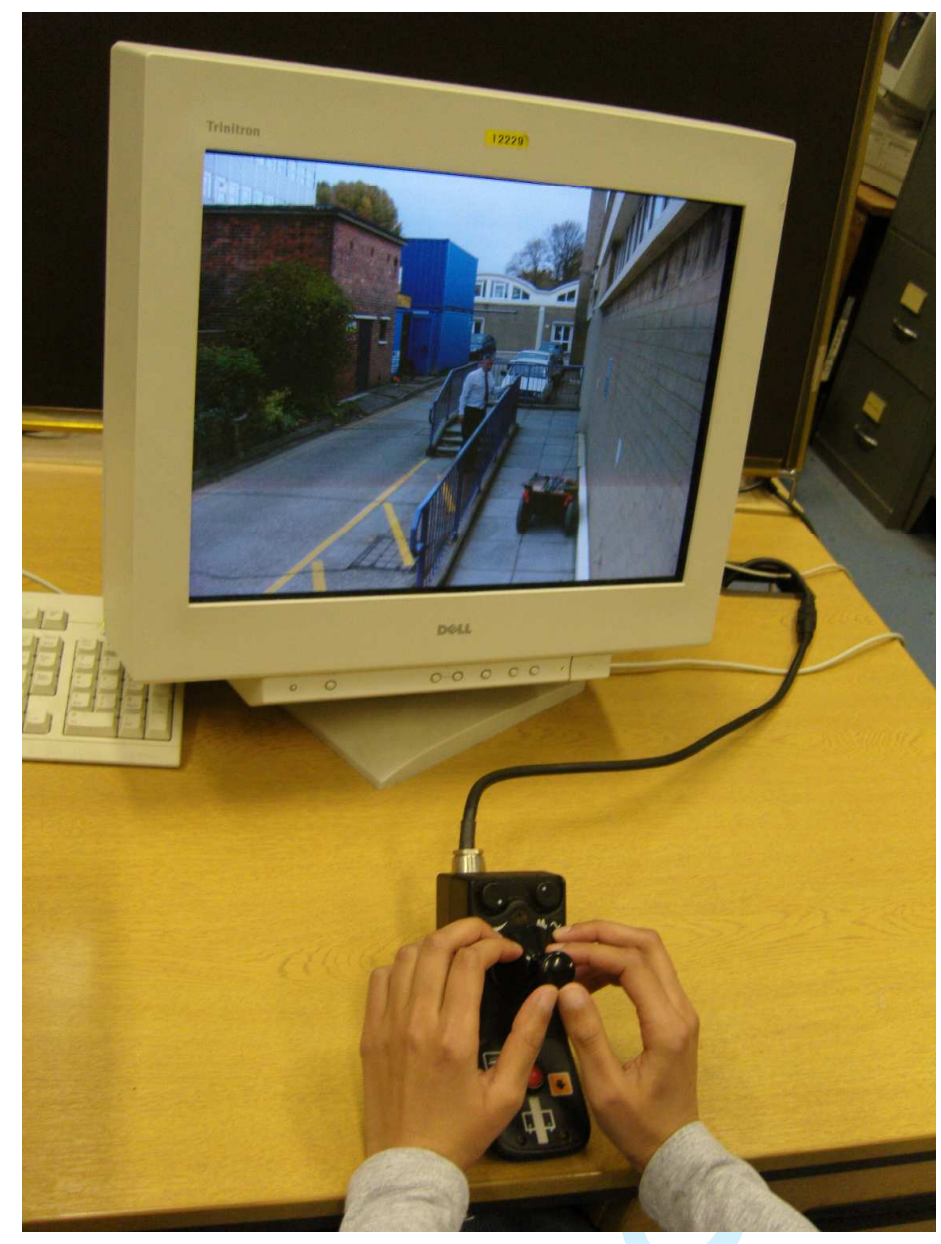

Figure $4 \mathrm{~A}$ tele-operator in a laboratory controlling the robot through an umbilical cable with a camera set up to observe the environment and the robot. 
Industrial Robot
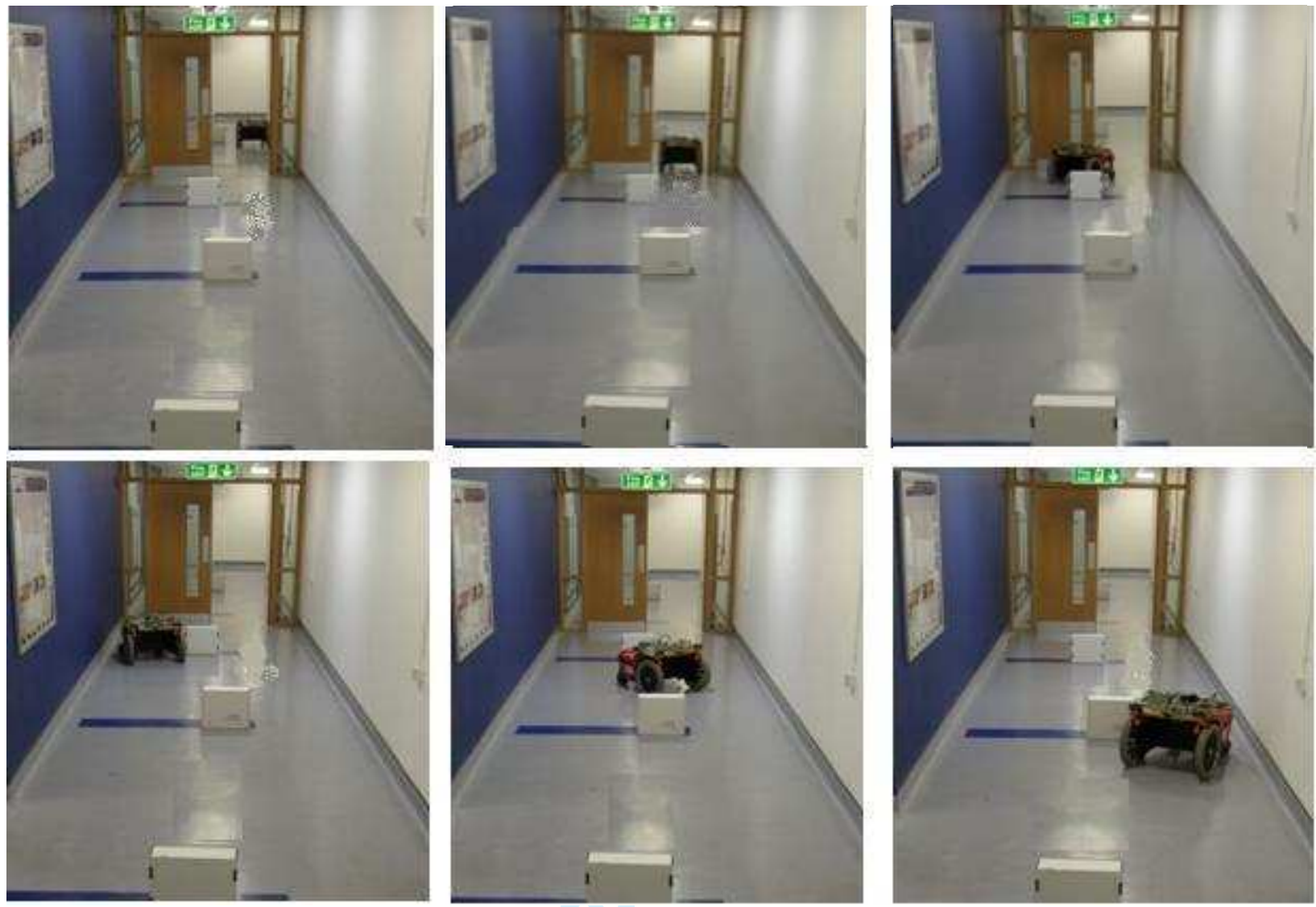

Figure 5 Radio controlled robot moving though a complicated corridor assisted by the sensor system on the mobile-robot. 
Page 29 of 38

Industrial Robot

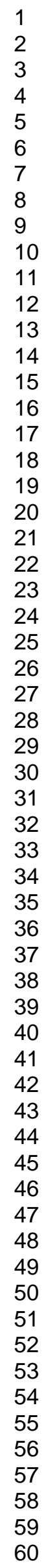



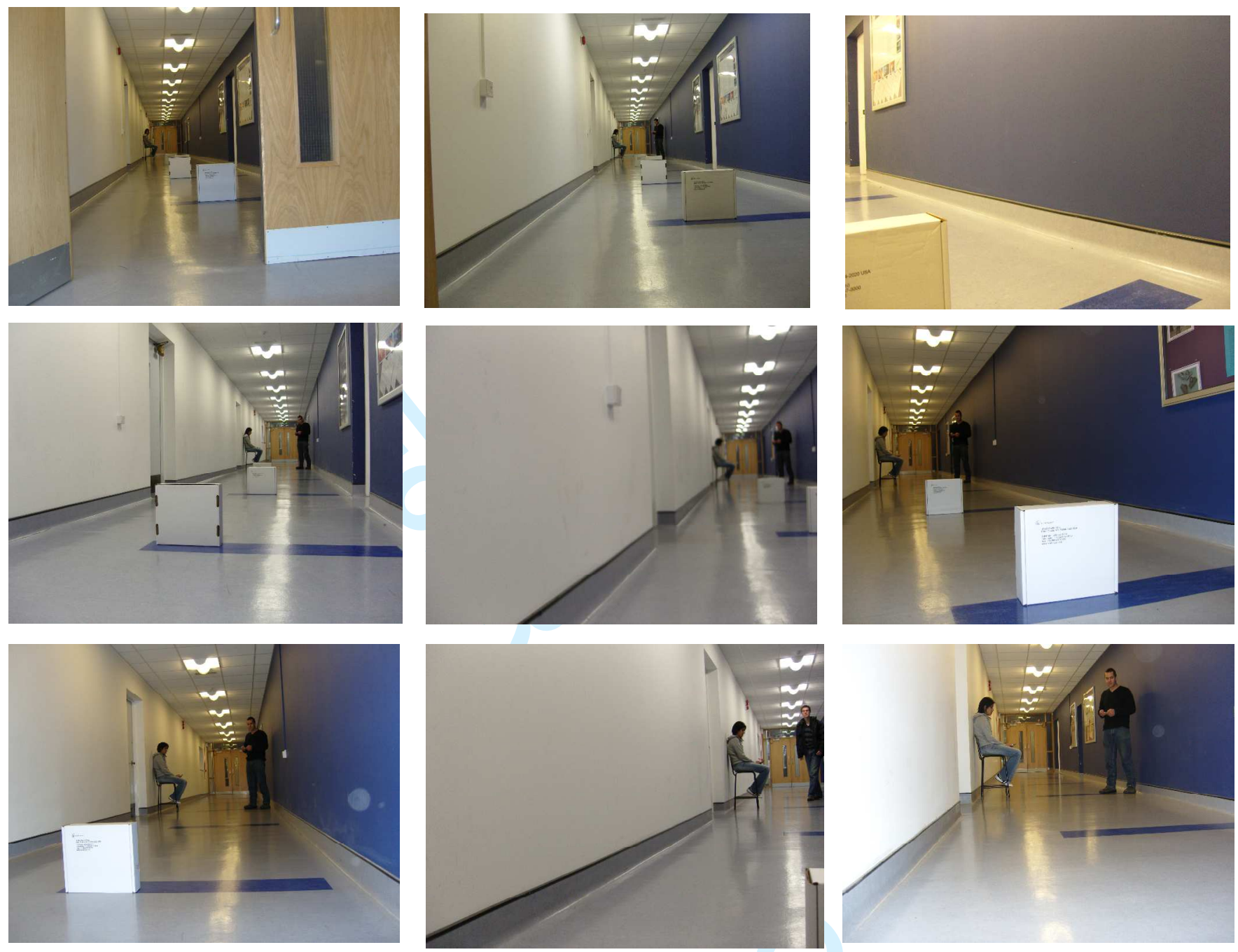

Figure 7 View from a camera mounted on the robot connected via an umbilical cable and moving though a complicated corridor while being assisted by the sensor system. 

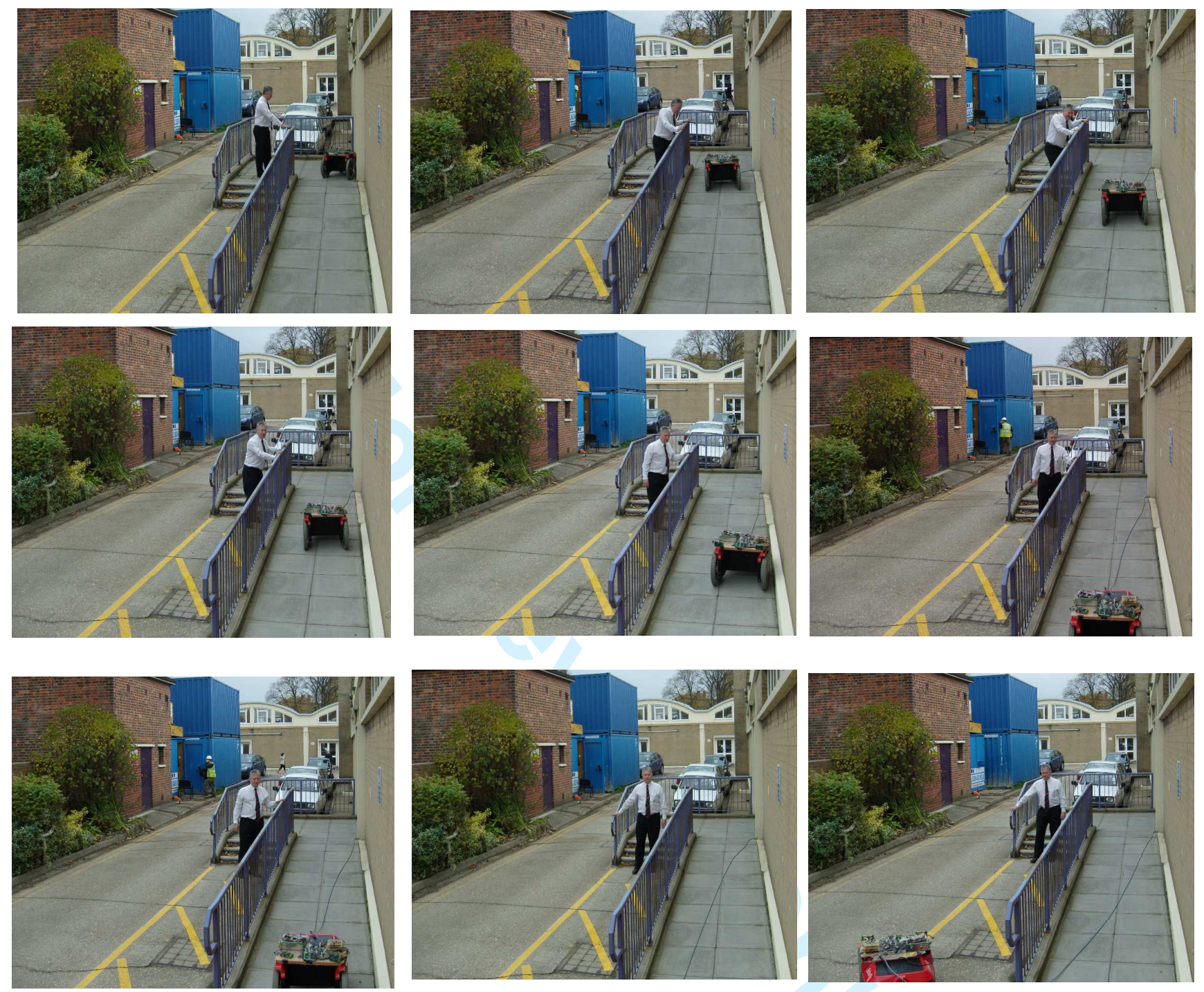

Figure 8 View observed by a tele-operator in a laboratory from an external camera while driving the robot though a complicated outdoor environment using an umbilical cable and assisted by the sensor system with a time delay of 0.5 seconds. 

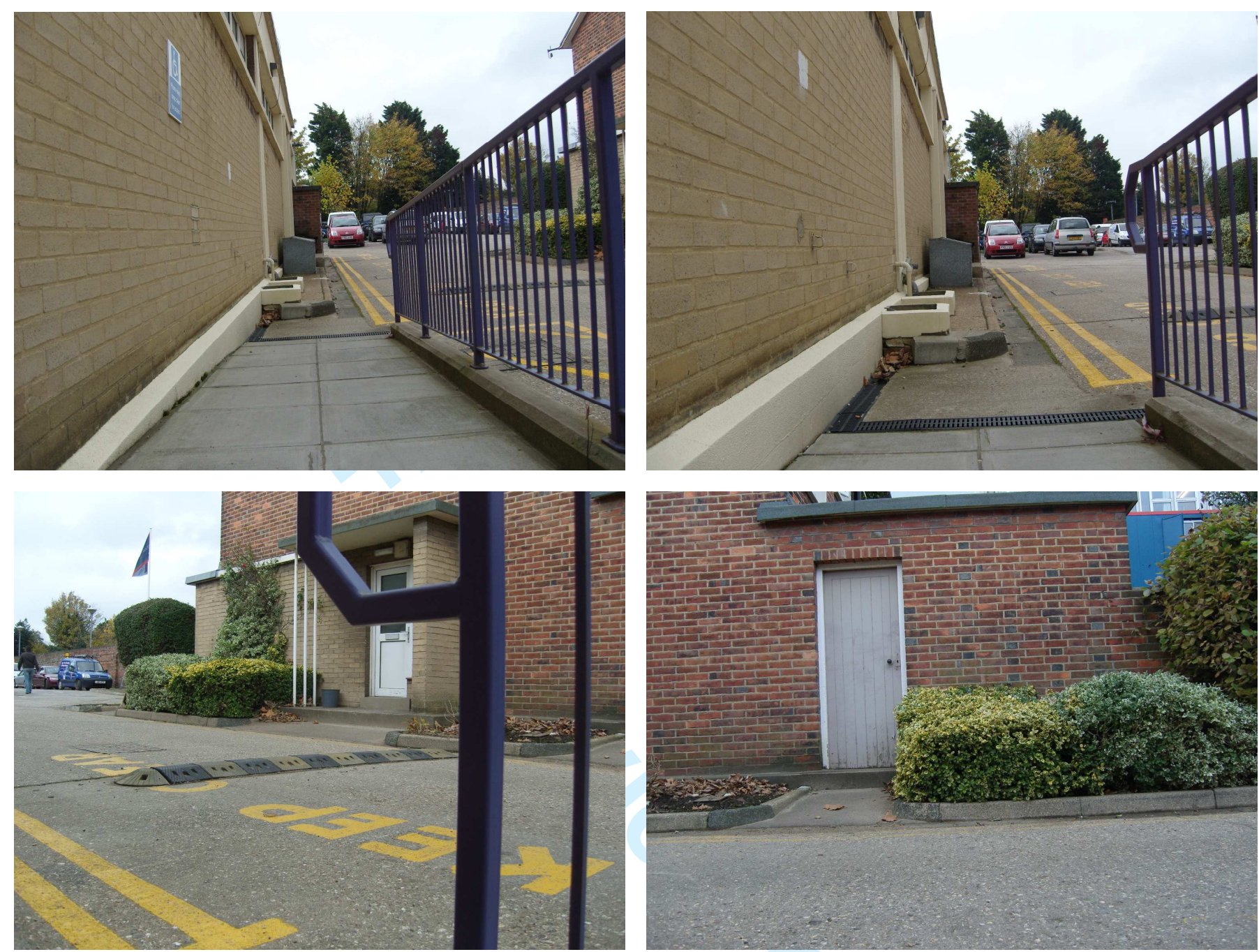

Figure 9 View from a camera mounted on the front of the mobile-robot moving though the first part of a complicated outdoor environment. With a delay of one second 

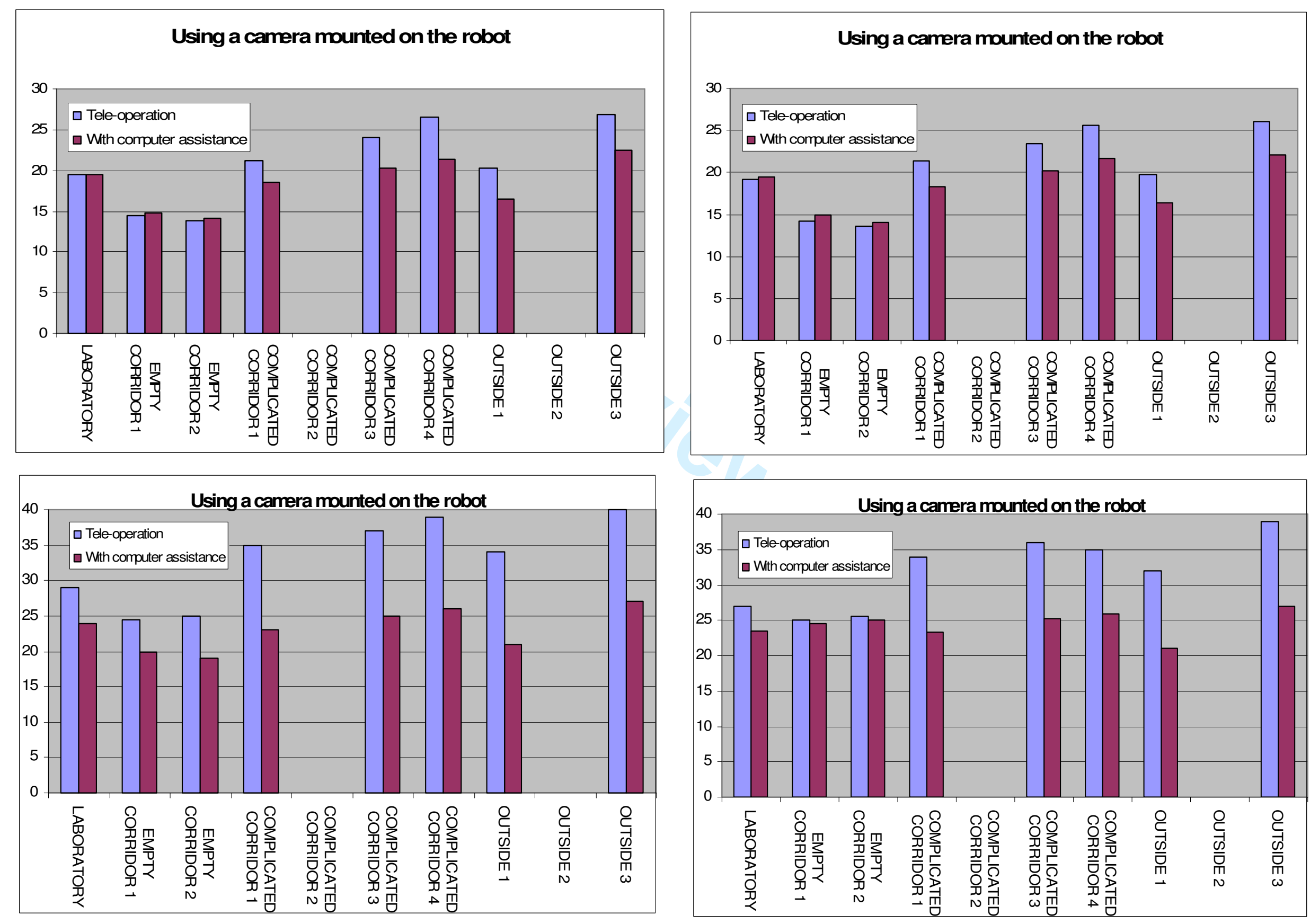
Industrial Robot

Page 34 of 38
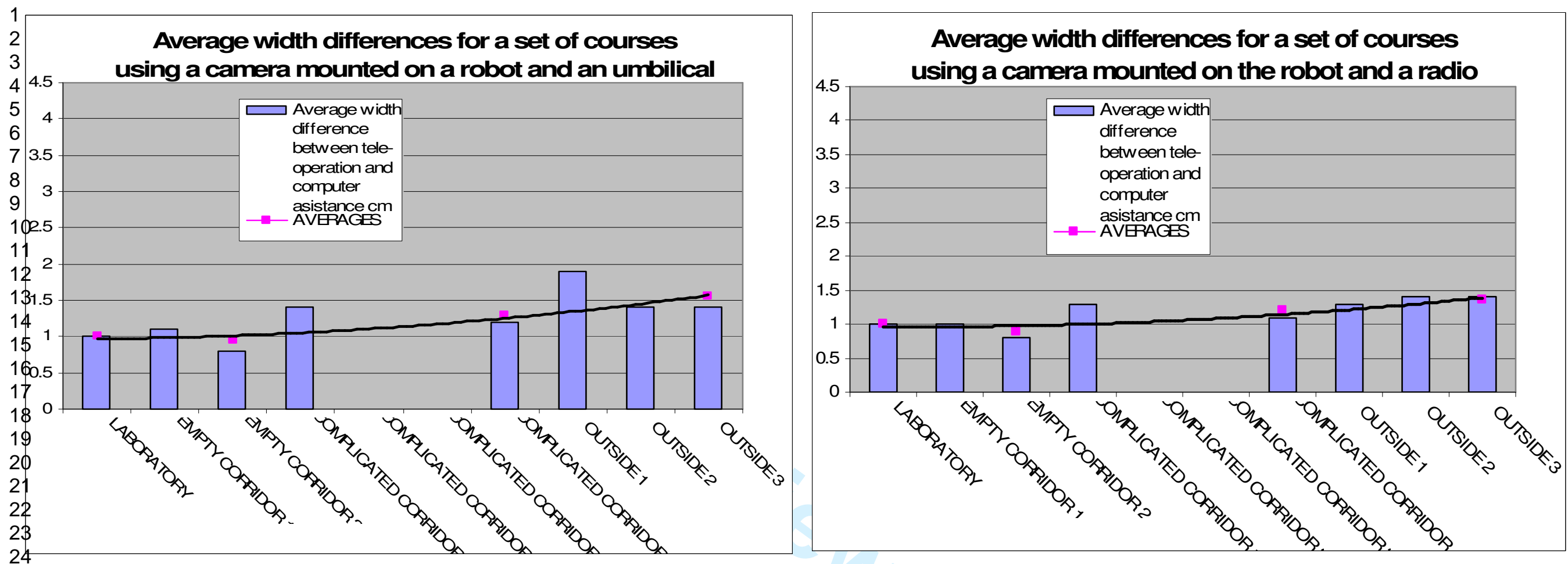

$26 \quad$ Average width differences for a set of courses

27 using a camera mounted on a robot and an umbilical

28

29

313.5

32

33 34 2.5

35

36 1.5

38

390.5

40

41

42

43

44

46

47

48

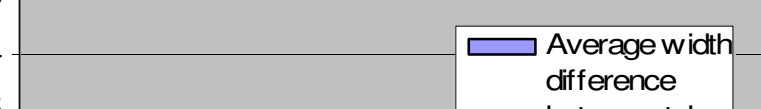

difference

operation and

computer

- assistance cm

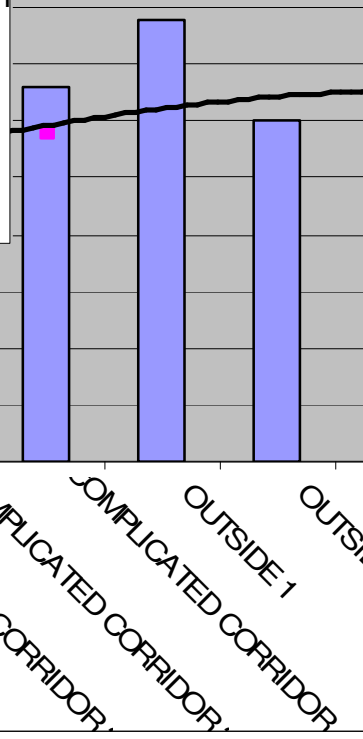

Average width differences for a set of courses using a camera mounted on the robot and a radio

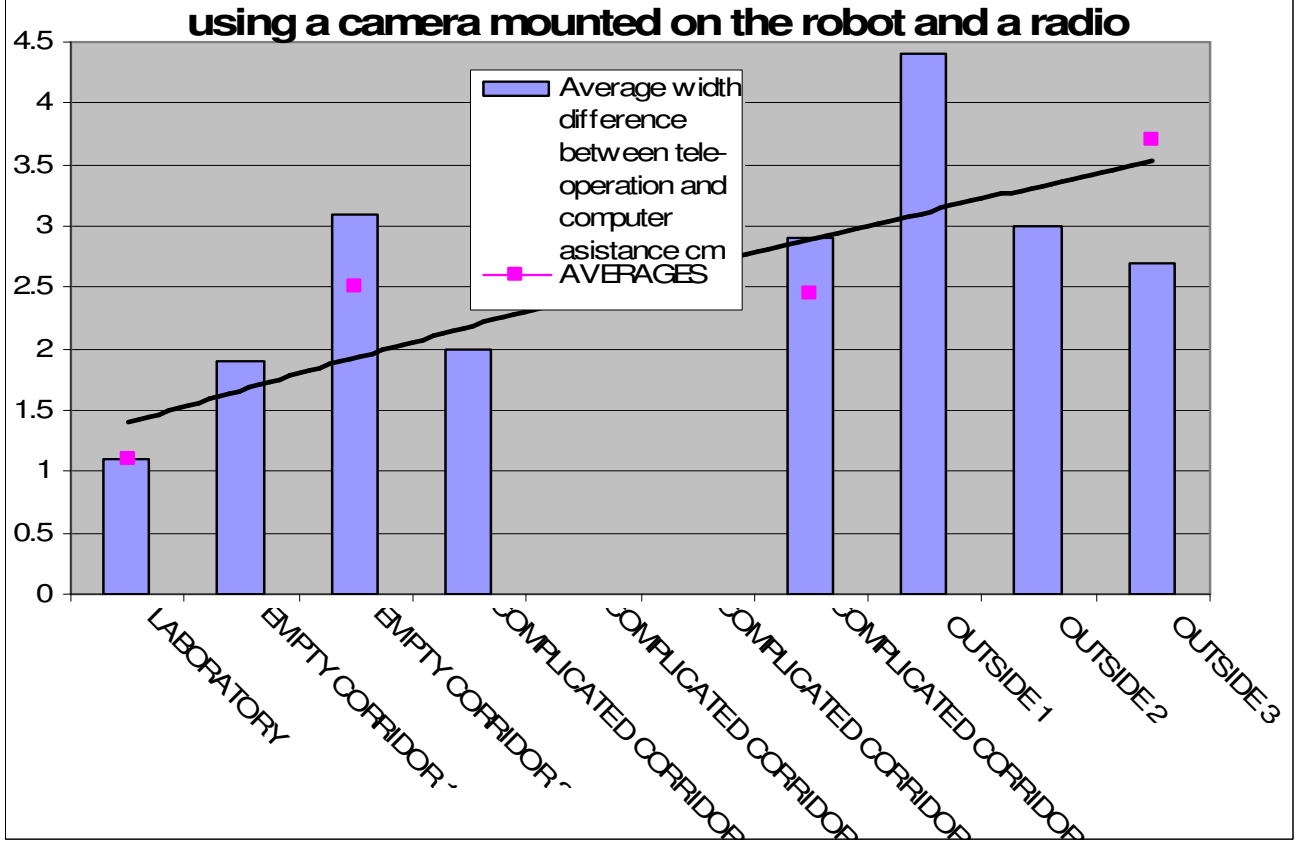




\section{Page 35 of 38}
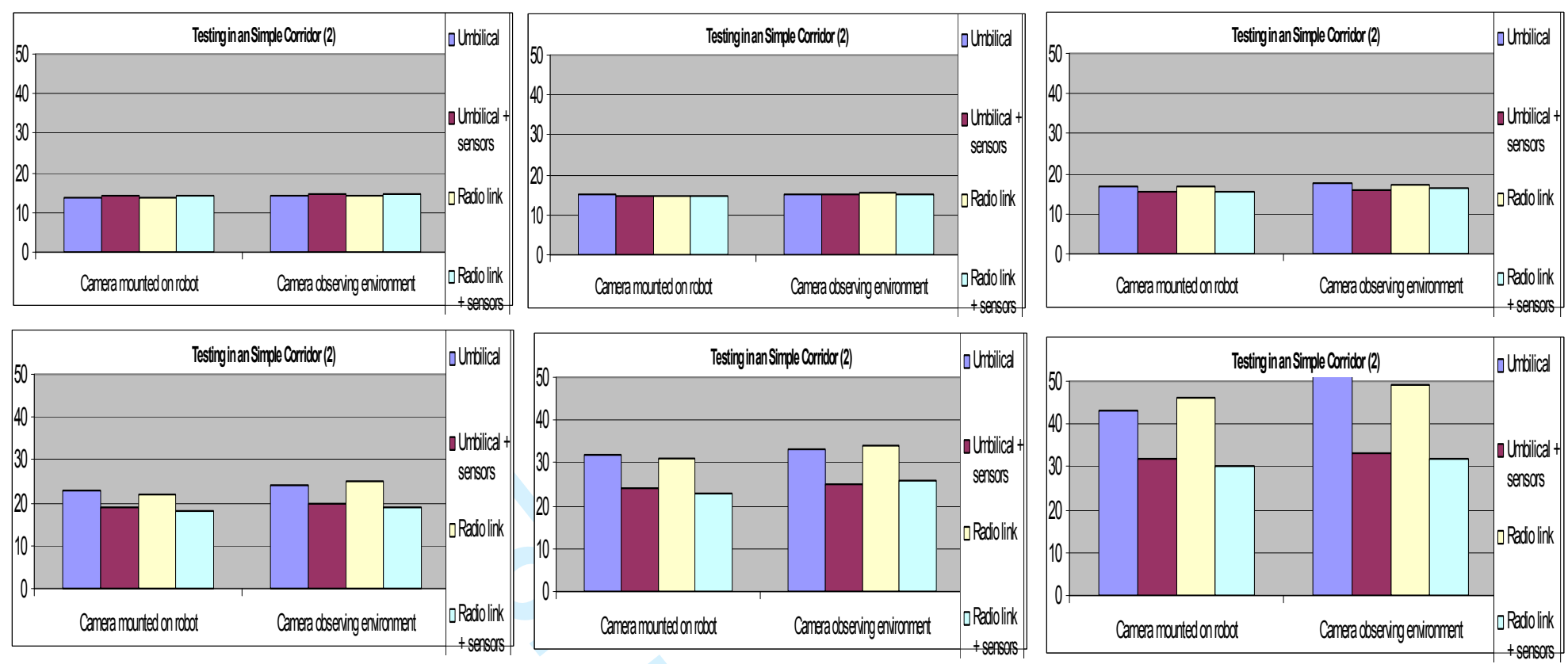

Figure 12 Results from testing in Simple Corridor 2 as the time delay was increased from real time (no delay) to 2.5 seconds in 0.5 second increments.
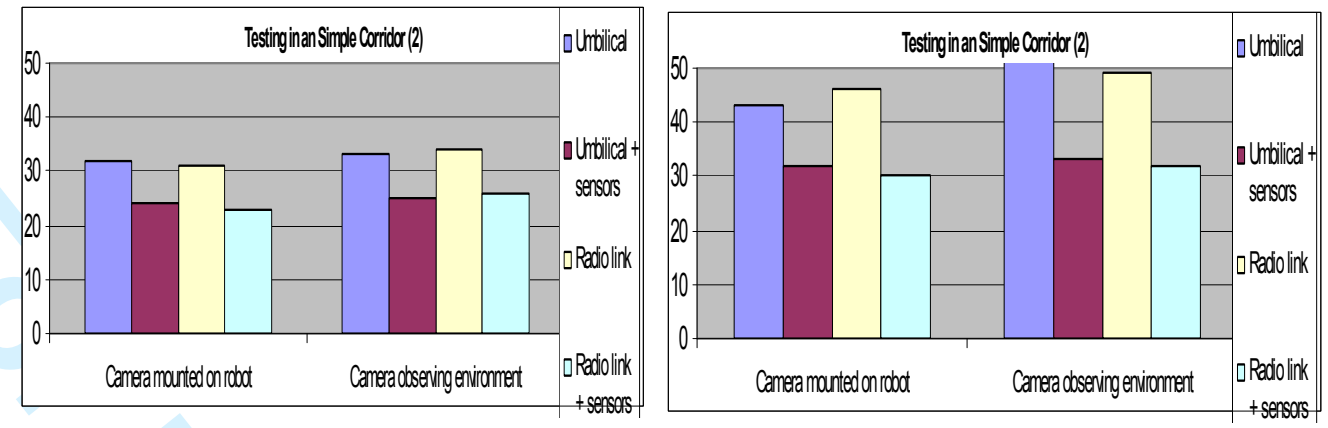

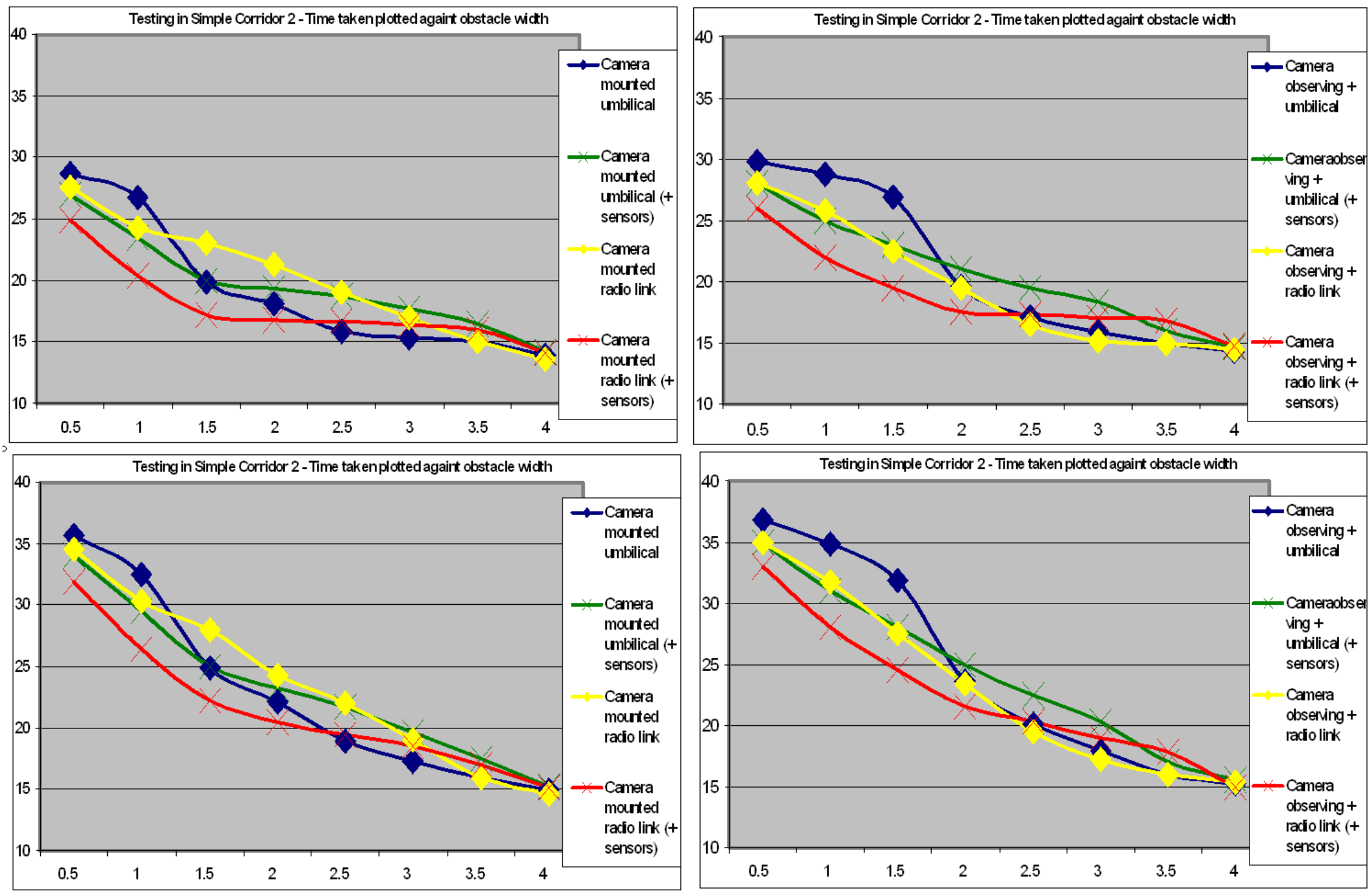

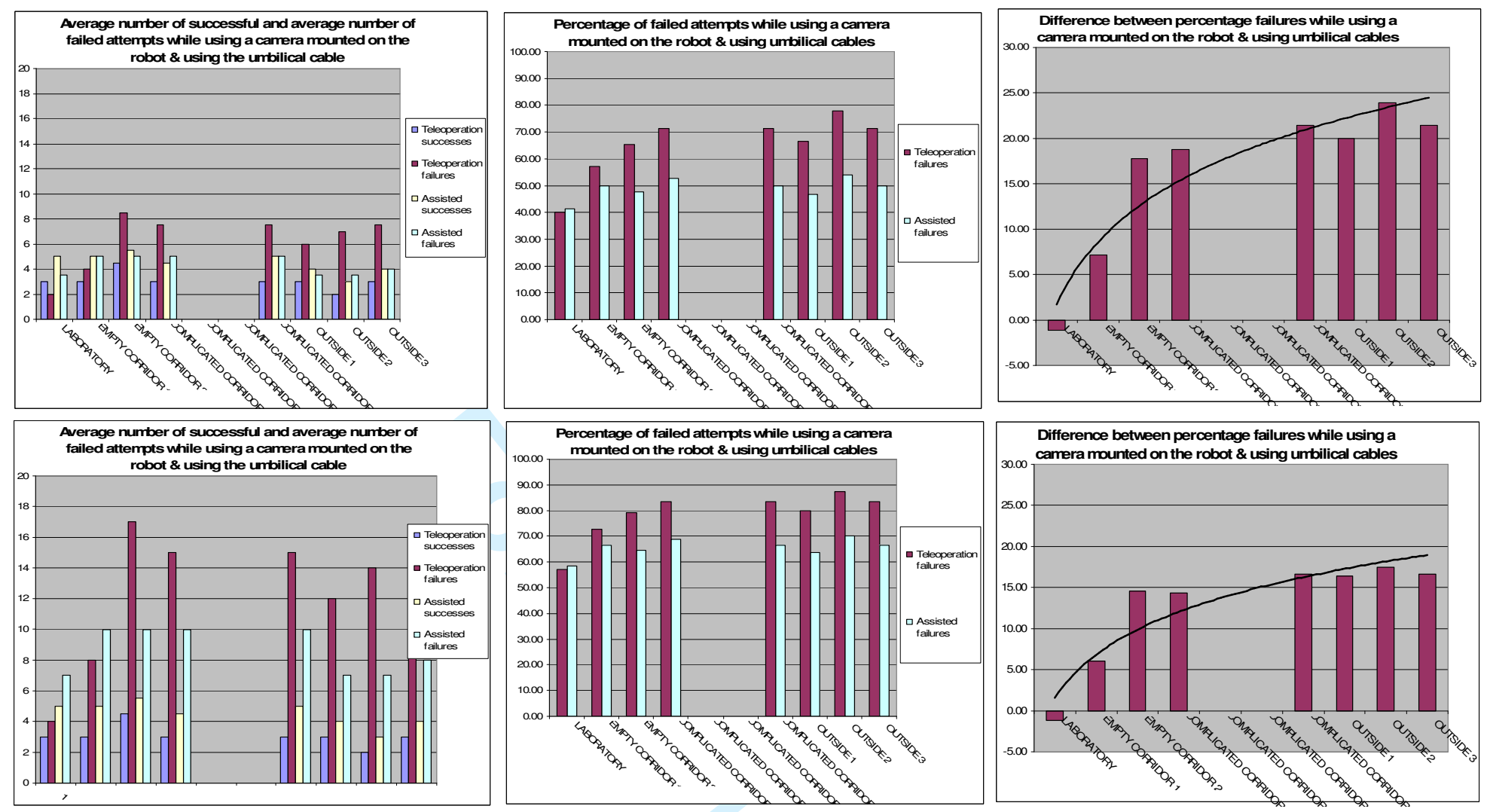

Figure 14 Comparison between average number of successful and average number of failed attempts with a time delay of one second introduced (below) while using a camera mounted on the robot and using an umbilical cable (left), percentage of failed attempts while using a camera mounted on the robot and using an umbilical cable (centre) and difference between the percentage of failed attempts with and without the sensor system while using a camera mounted on the robot and using an umbilical cable 


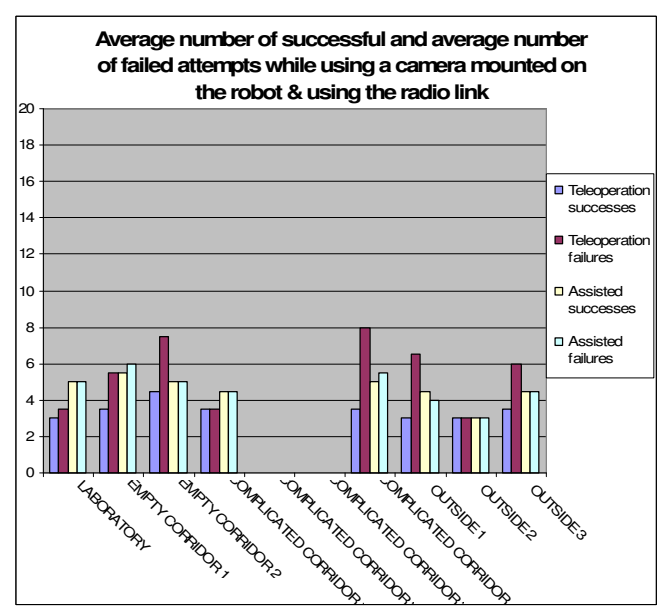

Industrial Robot
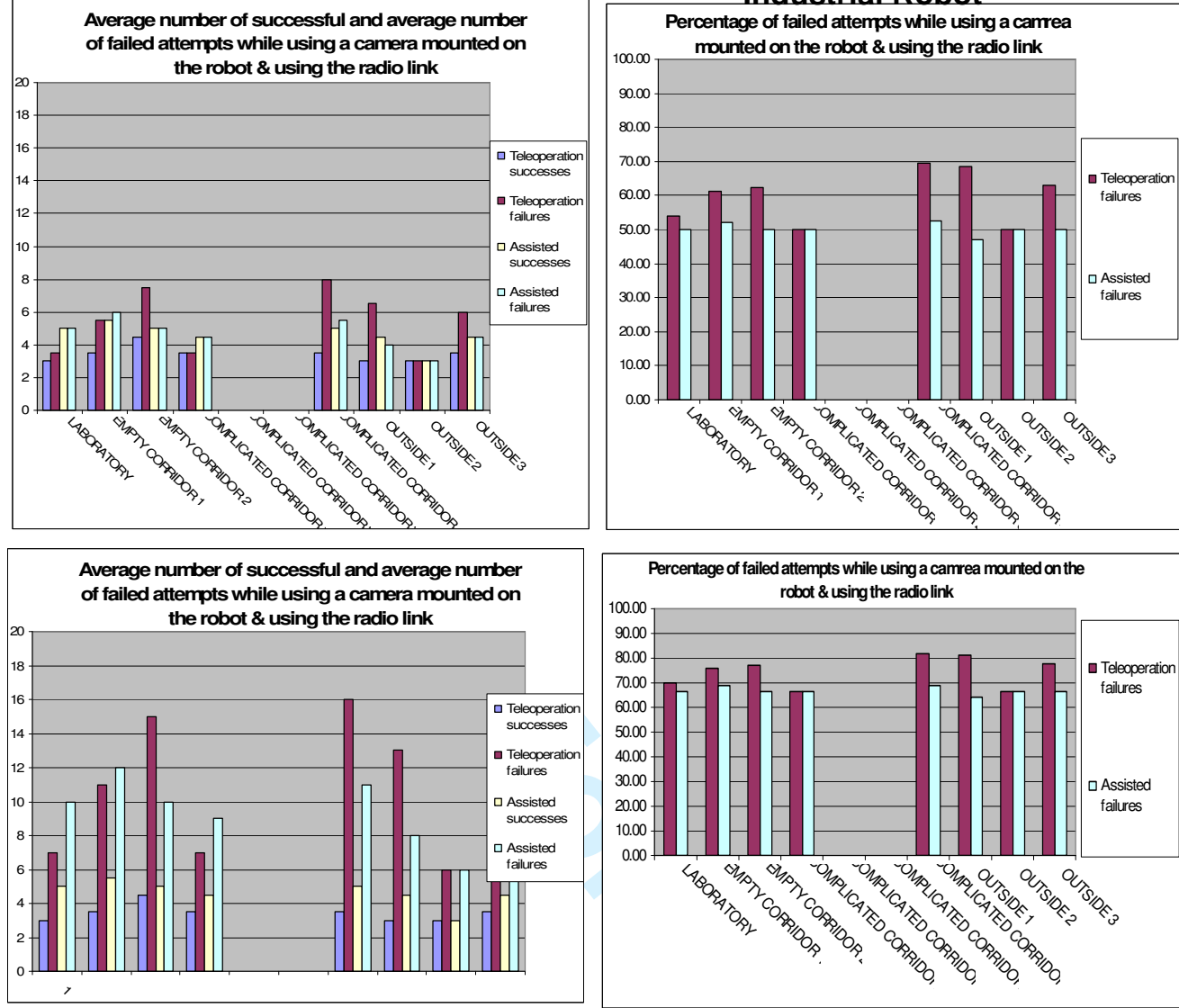

Page 38 of 38

Difference between percentage failures while using a
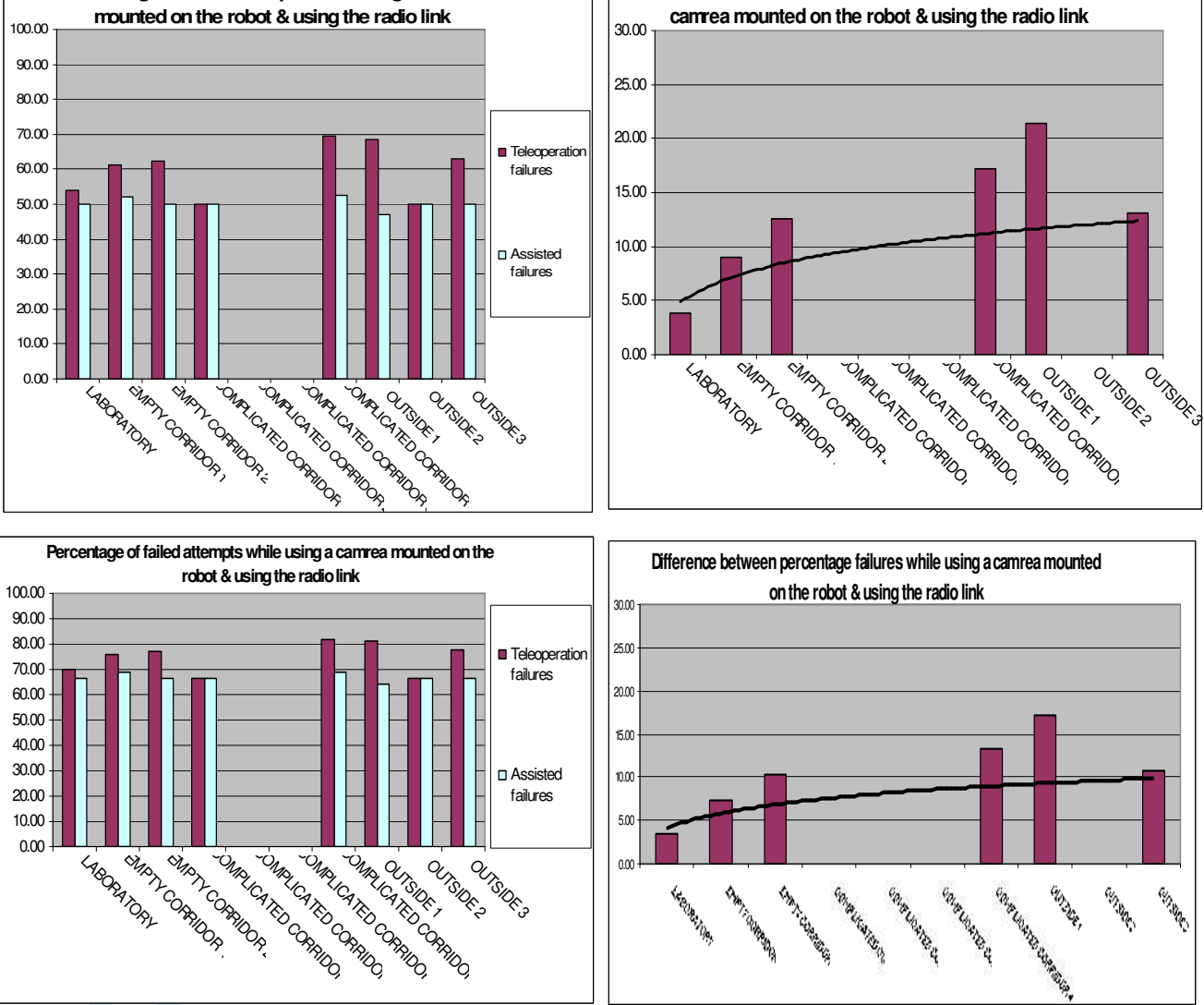

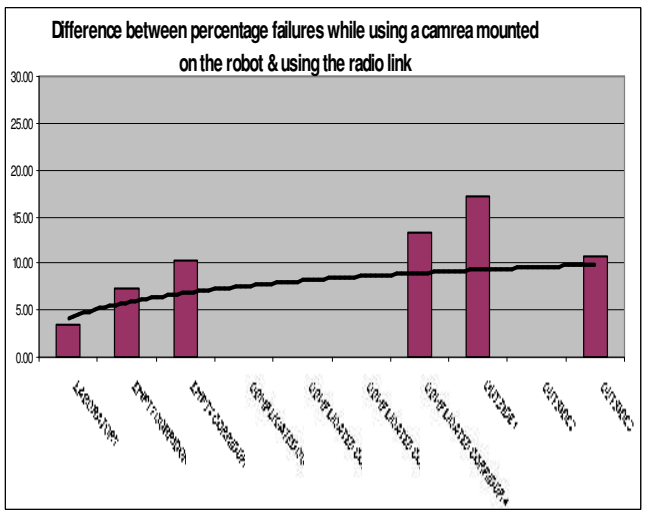

Figure 15 Comparison between average number of successful and average number of failed attempts with a time delay of one second introduced (below) while using a camera mounted on the robot and using a radio link (left), percentage of failed attempts while using a camera to observe the robot and using a radio link (centre) and difference between the percentage of failed attempts with and without the sensor while usng a radio link (right). 